\title{
A Model of Strategic TARgeted AdVERTISING
}

\author{
ANDREA GALEOTTI \\ JosÉ LUIS MORAGA-GONZÁLEZ \\ CESIFO WORKING PAPER NO. 1196 \\ CATEGORY 9: INDUSTRIAL ORGANISATION \\ MAY 2004 \\ Presented at CESifo AREA CONFERENCE ON IndUstrial ORganisation \\ MARCH 2004
}
An electronic version of the paper may be downloaded
- from the SSRN website:
- from the CESifo website: www.CESifo.de




\title{
A Model of Strategic TARGeted AdVERTISING
}

\begin{abstract}
We study a simultaneous move game of targeted advertising and pricing in a market with various consumer segments. In this setting we explore the implications of market segmentation on firm competitiveness. If firms are unable to target their ads on different consumer segments, a unique zero-profit equilibrium exists. By contrast, if firms employ targeted advertising, they can obtain positive profits. In equilibrium firms price very aggressively when they address the most profitable segment, quite gently when they target their ads on the least profitable segment and moderately aggressive when they advertise in the entire market.
\end{abstract}

JEL classification: D43, D83.

Keywords: segmentation, targeted advertising, oligopoly, price dispersion, price discrimination.

\author{
Andrea Galeotti \\ Tinbergen Institute \\ Econometric Institute \\ H17 - 15 Burg. Oudlaan 50 \\ 3062 PA Rotterdam \\ The Netherlands
}

\author{
José Luis Moraga-González \\ Erasmus University Rotterdam \\ Department of Economics \\ H7 - 17 Burg. Oudlaan 50 \\ 3000 DR Rotterdam \\ The Netherlands \\ moraga@few.eur.nl
}

We thank Mark Armstrong, José Hernández, Luis Ubeda, Maarten Janssen, Matilde Machado, Marco van der Leij, Otto Swank, Rob van der Noll, and Sanjeev Goyal for their comments. The paper has also benefited from presentations at Alicante, Amsterdam, Carlos III (Madrid), CORE (Belgium), Erasmus, CESifo Munich, Tilburg, UCL and at the EARIE 2003 Conference. 


\section{Introduction}

Markets are typically comprised of various consumer segments. For instance, there are settings where consumers are individual entities and thus each consumer represents a segment. In other settings, individuals have some unifying characteristics, such as mother tongue, and therefore can be grouped into distinct consumer segments. Other types of consumer segmentation are based on gender, geographical location, marital status, sexual orientation, etc. When markets are segmented, firms can typically decide to address one or more consumer segments as well as the entire market by choosing their advertising strategies appropriately. From the point of view of the firms, the question that arises is how they should market their products optimally.

As an example, consider consumer segmentation based on mother tongue. This type of segmentation is relevant in those countries with several official languages such as Belgium, Spain, Switzerland, etc. and is becoming important in other countries such as the US where the Spanish speaking community is sizeable. A firm operating, say, in the Belgium market may decide to enter only the French-speaking community and do so by inserting commercials in TV channels that broadcast only in French, or by inserting ads in newspapers and magazines written in French. Likewise, the firm may decide to address only the Dutch-speaking community and proceed by targeting its advertisements on these customers. Finally, the firm may want to address all the customers in the market rather than just one of the consumer segments. In this paper we develop a theory of competition in markets where firms can decide about the target of their advertising strategy.

We examine a simultaneous move game of pricing and advertising between firms selling a homogeneous product in a market consisting of various consumer segments. ${ }^{1}$ Consumers in all segments are initially uninformed about the firms' offerings and their prices and thus the firms' advertising decision represents the decision to target product and price information on them. In this setting, we explore the scope of the idea that market segmentation mitigates firm competitiveness. Even though segmentation has nothing to do with product differentiation here and thus consumers always buy from the lowest-price supplier, we show that it is sufficient to relax market competitiveness for some parameters.

\footnotetext{
${ }^{1}$ The assumption that products are homogeneous enables us to isolate the effects of segmentation. That is, in our setting consumers differ in attributes that are preference irrelevant.
} 
The basic features of the strategic game we study are that the population of consumers is segmented into various consumer groups and that firms advertising strategies can be designed to reach the distinct consumer segments and thus also the entire market. In the simplest version of our model consumers are 'perfectly' segmented into two groups $A$ and $B$ of possibly different size. Perfect segmentation refers to the situation where a consumer can only belong to a single segment, like in the case of segmentation based on gender. Firms may decide to target their ads on segment $A$ at a cost $\phi_{A}$ and charge a price $p_{A}$, or to target their ads on segment $B$ at a cost $\phi_{B}$ and charge a price $p_{B}$, or to advertise a price $p$ to all the consumers in the market at a cost $\phi_{A}+\phi_{B}$. The advertising costs should be seen as the cost of targeting product and price information on the different markets. In this model, we show that segmentation enables firms to make money in situations where they would only break even in its absence; this illustrates the idea that segmentation may relax market competitiveness. The reason is as follows: in the absence of segmentation, firms cannot be active in the market with probability one and thus they make zero profits. By contrast, when the market is segmented there exist parameters for which firms are always active in the market, yet the probability that they compete for the same consumers is relatively low.

In addition, our results characterize the nature of price-advertising equilibria in segmented homogeneous product markets. A natural way for firms to market products would be splitting the market so that each firm serves one of the segments. We show, however, that an equilibrium in pure strategies does not exist which implies that such segmentation equilibrium is not strategically viable. In equilibrium, firms randomize in pricing as well as in advertising strategy. In particular, firms randomize between targeting their ads on segment $A$, targeting their ads on segment $B$, and advertising to the entire market. Thus, the market outcome can exhibit segmentation but not with probability one. Further, we find that firms enter the most profitable segment more often than the least profitable one. Furthermore, firms price very aggressively when they go for the most profitable segment, quite gently when they target their ads on the least profitable segment and moderately aggressive when they advertise in the entire market. This random pricing and advertising strategy leads to positive profits provided that segments differ sufficiently either in size or in advertising cost. When the profitability of the distinct segments is similar instead, firms stay out of the market with positive probability and thus obtain zero profits in equilibrium. 
We now turn to discuss how parameter changes affect equilibrium outcomes. We focus on the positive-profits equilibrium described above. ${ }^{2}$ We start by examining the implications of an increase in advertising costs. We find that an increase in advertising fees makes it more likely that the market outcome exhibits segmentation: firms increase the probability with which they target their ads on the distinct consumer segments and decrease the probability with which they advertise in the entire market. Interestingly, this results in an increase in profits when the advertising cost is low initially, and in a fall in profits otherwise. The intuition behind this non-monotonic relationship between profits and advertising costs is as follows. An increase in the cost of advertising makes competition for the entire market a relatively unattractive strategy compared to competition for the segments. Since a firm must be indifferent between all three advertising strategies in equilibrium, firms decrease the frequency with which they compete for the entire market. This weakens competitiveness and results in an increase in revenues. When advertising costs are low initially, this revenue effect outweighs the cost effect and firms' profits rise. ${ }^{3}$ Finally, we observe that as advertising costs go to zero, the likelihood of segmentation converges to zero, the equilibrium distribution of prices charged in the entire market converges to a price distribution that is degenerate at the marginal cost and firms profits converge to zero. This is reminiscent of the Bertrand paradox.

We next study how an increase in the difference between advertising cost across segments influences the equilibrium. We find that firms profits are non-monotonic in this difference for some parameters. When the cost difference rises, firms increase the probability with which they target their ads on the most profitable segment, and decrease the probability with which they go for the entire market. This increases the likelihood of market segmentation. For large cost asymmetries, an increase in costs differences makes it very unattractive to advertise in the most expensive segment and firms end up competing very aggressively for just one of the consumer segments, which results in lower profits. If advertising costs asymmetries are small initially, firms benefit from an increase in fees differences because they compete for the entire market less often.

We examine a couple of extensions of the basic model. First we look at the implications of 'imperfect' consumer segmentation. To do this, we study a market where segments $A$ and $B$ overlap, that

\footnotetext{
${ }^{2}$ We note that the comparative statics results for the zero-profits equilibrium yield similar insights.

${ }^{3}$ The anti-competitive effects of increasing advertising costs have also been pointed out by Stahl (1994) in a model of (non-targeted) advertising. The mechanism here is different because firms enter the market with probability one.
} 
is, some consumers belong to both segments. In the example of segmentation based on mother tongue, this set comprises those consumers who are bilingual. We show that the equilibrium of the benchmark model discussed above survives this change but we find that imperfect segmentation reduces the set of parameters for which this equilibrium exists. What is more interesting is that firms may benefit as segmentation becomes more imperfect. Indeed, we find that when the market is imperfectly segmented, other equilibria with positive profits can be sustained. In a second extension of the basic model, we allow for entry of firms. We show that in the pricing and advertising equilibrium described above profits decline as the number of firms in the market rises.

The last issue we discuss is what happens if firms can simultaneously advertise a price to segment $A$ 's consumers and a different price to segment $B$ 's consumers. Even though there is no consumer heterogeneity in our model, a firm has incentives to price discriminate in our model to strengthen its undercutting power. As a result, we find that price discrimination erodes all possibilities to obtain economic profits. This result provides another instance where competing firms benefit from bans on price discrimination. ${ }^{4}$

Our paper should be seen as a contribution to the study of advertising in oligopolistic industries. The novelty of our approach is that we examine the strategic use of targeted advertising in homogeneous product markets. The literature on informative advertising has in general ignored the possibility that firms can choose to target their offers on distinct consumer groups. ${ }^{5}$ An exception is the monopoly model of Esteban et al. (2001), who show that a firm use of specialized magazines as a vehicle to target price advertisements on the consumers who value the good more leads to higher advertised prices. The only papers we know where oligopolistic firms can choose to target their ads on distinct buyer groups are Iyer et al. (2002) and Roy (2000). The first paper examines a model where products are differentiated. Some consumers are loyal to one firm, some other consumers are loyal to the other firm and the rest of the consumers are price-sensitive. Seen from the viewpoint of our work, loyalty gives firms reasons other than strategic to employ targeted advertising. Roy

\footnotetext{
${ }^{4}$ The literature on price discrimination and imperfect competition is surveyed in Stole (2003). Holmes (1989) is an example where firms obtain higher profits if price discrimination is not allowed. Armstrong and Vickers (2001) show that this result is reversed if markets are sufficiently competitive. Thisse and Vives (1988) analyze a spatial model where firms profits are lower under location-based pricing than under uniform pricing.

${ }^{5}$ See e.g. Bester and Petrakis (1995), Butters (1977), Grossman and Shapiro (1984), Stahl (1994), and Stegeman (1991).
} 
(2000) studies a two-stage model where firms first send product-advertisements to the consumers and then choose their prices. Advertising has a long-run nature and a commitment to not invade the 'natural' market of the rival enables firms to segment the market and appropriate consumer surplus. His model applies to markets where advertising provides product information -and not price information-, perhaps intended to create brand image and consumer awareness, and, later, buyers discover prices costlessly. By contrast, our paper is in line with the main bulk of the advertising literature and examines firm competition in environments where advertising has a short-run nature and conveys price information.

Our paper develops a theory of competition in markets that are segmented and where firms offer truly homogeneous products. The key point in our paper is that here segmentation is unrelated to consumer preferences and this enables us to isolate its effects on competition. We show that this case of minimal segmentation is sufficient to mitigate firm competitiveness. In this sense, this paper is complementary to the body of work that has examined competition in markets with customer loyalty (see e.g. Rosenthal, 1980), and in truly product differentiated markets, either with respect to vertical attributes (see e.g., Shaked and Sutton, 1982) or with respect to horizontal attributes (see e.g., d'Aspremont, Gabszewicz and Thisse, 1979). This work has produced the standard result that product differentiation relaxes price competition between firms.

The remainder of the paper is organized as follows. Section 2 describes the basic model. Section 3 presents the equilibria, the comparative statics results and some extensions of the basic model. Section 4 discusses price discrimination. Section 5 closes the paper with a review of the main conclusions. All proofs are relegated to the Appendix.

\section{The model}

We examine a simultaneous advertising and pricing game between homogeneous product sellers. The crucial feature of the setting we study is that the consumer market is segmented. As discussed in the Introduction, we assume here that firms market homogeneous products, which implies that segmentation is based on utility-irrelevant attributes. ${ }^{6}$ Examples of such attributes include gender,

\footnotetext{
${ }^{6}$ This assumption, along with others that will appear later, implies that we focus on a case of extreme competition. The main idea of this paper, namely, that targeted advertising help firms weaken competitiveness should however be
} 
mother tongue, marital status, cultural interest, sexual orientation, geographical location, level of education, etc. On the demand side of the market, there is a set of consumers who hold downward sloping demand functions $D(p)$. For later reference, we define the revenue per consumer as $R(p)$ and assume that $R^{\prime}(p)>0$, for all $p \in\left[0, p^{m}\right]$, where $p^{m}=\arg \max _{p} R(p)$. Let $R^{-1}(\cdot)$ be the inverse of the revenue function. For our purposes, it will be enough to assume that consumers can be grouped into two market segments $A$ and $B$, with sizes $\mu_{A}$ and $\mu_{B}$ respectively, $\mu_{j}>0, j=A, B$. We normalize the total number of consumers to 1 ; thus $\mu_{A}+\mu_{B}=1 .^{7}$

On the supply side of the market there are $N \geq 2$ firms. These firms produce the good at constant returns to scale and we normalize the marginal cost of production to zero without loss of generality. Consumers ignore, a priori, the existence and the price of the products so that firms must inform them to be able to sell. A firm $i$ may decide to address either the consumers in segment $A$, or in segment $B$, or in both segments $A$ and $B$ (i.e., the entire market), or, finally, stay out of the market altogether. ${ }^{8}$ We shall represent this set of pure advertising-strategies as $E_{i}=\{O, A, B, M\}$, where $O$ denotes the decision to stay out of the market and $M$ indicates the decision to send ads to all the consumers in the market. A firm $i$ 's mixed advertising-strategy is then a probability function over the set $E_{i}$. We will refer to $\lambda_{j}^{i}$ as the probability with which a firm $i$ sends its ads to market $j \in E_{i}$. We assume that firms face an advertising cost $\phi_{j}>0$ to address consumer segment $j, j=A, B .{ }^{9}$ Clearly, a firm sending its ads to the entire market bears a total cost of $\phi_{A}+\phi_{B} \cdot{ }^{10}$ To make the problem interesting, we assume that $\phi_{j}<\mu_{j} R\left(p^{m}\right), j=A$, B, i.e., each segment is worth at monopoly price. We will refer to the ratio $\phi_{j} / \mu_{j}$ as the profitability of segment $j, j=A, B$.

valid in other environments, for instance, where products are differentiated.

${ }^{7}$ This specification implies that the market is perfectly segmented in the sense that if a consumer belongs to a segment then he/she does not belong to the other segment. Segmentation based on gender is an example of perfect segmentation. As argued in the Introduction, sometimes segmentation is imperfect, like segmentation based on mother tongue. Geographical segmentation is also typically imperfect. For example, consider Rotterdam city as a market; the city is divided by the Maas river into the north side and the south side but there are consumers who commute for a number of reasons. In section 4 we show that our main results extend to imperfectly segmented markets.

${ }^{8}$ We are thus assuming not only that firms can target their ads on a segment as well as on the entire market but also that they can choose which segment to address. If consumers are atomistic, or if segmentation is geographical, this is straightforward to do. If segmentation is based on some other attributes such as mother tongue sellers can easily address a group of buyers who speak a particular language by inserting ads in newspapers and magazines written in that language, or by inserting commercials in TV channels that only broadcast in that language.

${ }^{9}$ We are thus assuming that if a firm targets its ads on a particular market, this firm reaches all consumers in that market. This is certainly a simplifying assumption that enable us to solve the problem we are interested in. The fact that information provision is assumed to be perfect contributes to make our model a case of extreme competition.

${ }^{10}$ The nature of our results does not change if there are economies or diseconomies of scale in advertising (see footnote 14 below). 
We assume, without loss of generality, that $\phi_{A} / \mu_{A} \leq \phi_{B} / \mu_{B}$; this implies that, from the point of view of the firms, segment $A$ is more attractive than segment $B$, ceteris paribus. This will have interesting implications on the nature of the equilibrium advertising and pricing decisions.

For advertising decision $j \in\{A, B\}$, a firm $i$ 's pricing-strategy is denoted by a distribution of prices $F_{j}^{i}(p)$. When $j=M$, we consider two price scenarios. One, firms advertise a uniform price in the entire market so firm $i$ price is denoted $F_{M}^{i}(p)$. Two, firms advertise different prices in distinct consumer segments in which case we denote firm $i$ 's price as $\left\{F_{M, A}^{i}(p), F_{M, B}^{i}(p)\right\} .{ }^{11}$ Let $\sigma_{j}^{i}$ denote the support of $F_{j}^{i}(p)$ and let $\bar{p}_{j}^{i}$ and $\underline{p}_{j}^{i}$ denote the maximum and the minimum price in $\sigma_{j}^{i}$, respectively. A firm $i$ 's strategy is thus denoted by a collection of pairs $s^{i}=\left\{\left(\lambda_{j}^{i}, F_{j}^{i}(p)\right)\right\}_{j \in E_{i}}$.

Our interest lies on the existence and characterization of symmetric Nash equilibria. ${ }^{12}$ A symmetric Nash equilibrium is an advertising and pricing strategy profile such that an individual firm maximizes profits taking as given the strategies of the other firms.

\section{Analysis}

Our objective is to examine the influence of consumer segmentation and targeted advertising on the functioning of the market. We shall show that segmentation can be exploited by the firms to soften competition and that this has interesting implications on the nature of the market equilibrium. To see this, we will first examine the benchmark case of a market that is not segmented and show that in the unique symmetric equilibrium of the pricing and advertising game described above firms obtain profits equal to zero. Then we will study a perfectly segmented market and show that segmentation leads to a positive-profits equilibrium provided that firms do not advertise different prices at the same time. These issues are better explained by considering a duopolistic model so we will set $N=2$ in what follows. Later in this Section we show that our main result extends easily

\footnotetext{
${ }^{11}$ Some remarks on the issue of price discrimination are necessary here. In some settings price discrimination is certainly unfeasible. For instance, in line with the examples above, legal restraints typically imply that a firm cannot discriminate between persons of different sexual orientation, nor between men and women or between French-speaking and Dutch-speaking people. Sometimes price discrimination is legal but yet impractical. For example, when a shop has a single point-of-sale advertised prices must equal on-the-shop prices; in addition, it does not seem common practice to charge consumers different prices in the shop just because they have seen different ads. However, there may be contexts where price discrimination is feasible and practical and that is why, in Section 4, we explore the role that price discrimination has in our model.

${ }^{12}$ We also examine all pure asymmetric entry-strategy profiles (see Lemma 1 below).
} 
to a $N$-firm oligopoly game, as well as to the case of imperfect segmentation. In Section 4 , we shall consider the case of price discrimination.

\section{Non-segmented markets:}

As a benchmark case, we examine here a setting where firms are unable to target their ads on the consumer segments, that is, they can either advertise to the entire market or not at all, i.e., $E_{i}=\{O, M\}$. The following result, due to Sharkey and Sibley (1993), shows that the advertising and pricing game described above has a unique symmetric equilibrium in which firms obtain zero profits.

Theorem 1 In the unique symmetric equilibrium of the game firms stay out of the market with probability $\lambda_{O}=\frac{\phi_{A}+\phi_{B}}{R\left(p^{m}\right)}$ and enter the entire market with probability $\lambda_{M}=1-\lambda_{O}$ in which case they advertise a price $p$ randomly chosen from the set $\sigma_{M}=\left[R^{-1}\left(\phi_{A}+\phi_{B}\right), p^{m}\right]$ according to the price distribution $F_{M}(p)=1-\frac{1}{\lambda_{M}}\left(\frac{\phi_{A}+\phi_{B}}{R(p)}-\lambda_{O}\right)$. This equilibrium exists always.

The proof is sketched in the Appendix. We note that firms cannot be active in the market with probability one because competition would drive revenues down to zero. In equilibrium, firms must randomize between staying out of the market and advertising in the entire market, which yields zero-profits.

\section{Segmented markets:}

To examine the implications of targeted advertising in the presence of consumer segmentation, we analyze equilibria where firms can also decide to target their ads on a single segment, i.e., $E_{i}=\{O, A, B, M\}$. Our first remark is that a pure strategy equilibrium does not exist in this game either.

Lemma 1 A pure advertising-strategy cannot be part of an equilibrium.

We note that this result holds for symmetric as well as asymmetric pure strategies. Symmetric advertising-strategies are readily ruled out by noting that an individual firm always has an incentive to deviate, either by targeting its ads on non-served segments, or by exiting the market altogether. A further elaboration of these arguments shows that asymmetric advertising-strategy profiles cannot 
be part of an equilibrium either. The economic implication of this result is that the market outcome cannot exhibit 'permanent' segmentation in the sense that different consumer groups are always served by distinct firms. ${ }^{13}$ This contrasts with Roy (2000) and the difference in results stems from the long-run nature of advertising in his model.

We are now ready to present our main result: if firms can target their ads on different consumer segments, then firms can obtain positive-profits in equilibrium. We develop this result in two steps. We first examine the necessary conditions for a positive-profits equilibrium to exist. We then construct an equilibrium in which firms obtain positive profits and examine the region of parameters for which this equilibrium exists. Interestingly, positive profits require the segments to differ sufficiently either in size or in advertising costs.

Proposition 1 If a positive-profits equilibrium exists, then (a) $\lambda_{j} \in(0,1), j=A, B, M$, and $\lambda_{O}=0,(b) F_{A}(p), F_{B}(p)$ and $F_{M}(p)$ are atomless price distributions and (c) $\underline{p}_{A}<\bar{p}_{A}=\underline{p}_{M}<$ $\underline{p}_{B}<\bar{p}_{B}=\bar{p}_{M}=p^{m}$.

The proof, which is in the Appendix, proceeds as follows. We first prove that firms must advertise in the entire market with strictly positive probability; otherwise a firm obtaining positive profits would gain by deviating and sending ads to the entire market. Then we note that firms must allocate positive probability to targeting their ads on segment $A$ as well as on segment $B$; otherwise, a firm advertising in the entire market would gain by deviating and saving the cost of sending ads to one of the segments. The interpretation of these two remarks is that a positive-profits equilibrium requires firms to introduce quite a bit of noise in regard to their advertising strategies. We then show that there cannot be a single price common to all three equilibrium supports $\sigma_{A}, \sigma_{B}$ and $\sigma_{M}$ in a positive-profits equilibrium; otherwise a firm charging such price would not be indifferent between all three advertising strategies. The next step is to show that firm pricing behavior in every market is characterized by atomless price distributions and this follows from simple undercutting arguments. This tells us that only by obscuring the market sufficiently, firms can hope to make money in equilibrium. After this, a series of claims proves that the support configuration must satisfy the inequality above. These claims exploit two facts: first, that a firm that advertises in a

\footnotetext{
${ }^{13}$ To be more precise, for this claim to be true, one needs to prove that the (asymmetric) entry-strategy profiles $\lambda_{O}^{1}+\lambda_{A}^{1}=1$ and $\lambda_{O}^{2}+\lambda_{B}^{2}=1$ cannot be part of an equilibrium, which is straightforward to verify.
} 
single segment cannot increase its profits by deviating and entering the entire market; second, that a firm that advertises in the entire market cannot increase its profits by deviating and targeting its ads on a single segment.

To illustrate, the support configuration derived in Proposition 1,c) is depicted in Figure 1.

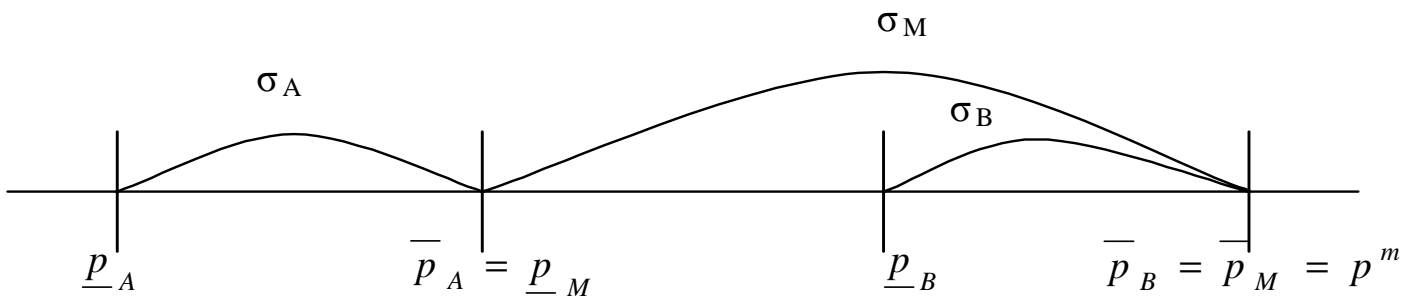

Figure 1: Configuration of price supports in a positive-profits equilibrium.

This Proposition shows that the maximum price firms charge when they target their ads on segment $A$ is strictly lower than the minimum price firms charge when they go for segment $B$. The reason for this result is related to the following inequality that compares the ex-ante profitability of different advertising strategies:

$$
\frac{\phi_{A}}{\mu_{A}} \leq \frac{\phi_{A}+\phi_{B}}{\mu_{A}+\mu_{B}} \leq \frac{\phi_{B}}{\mu_{B}}
$$

We note that segment $A$ is more attractive than segment $B$ because advertising cost per consumer is lower in the former than in the latter. In equilibrium, firms must be indifferent between advertising in segment $A$ and advertising in segment $B$, which can only be possible if the expected revenue per consumer in segment $A$ is lower than in segment $B$. One way to accomplish this is to announce high prices in segment $B$ and low prices in segment $A .^{14}$

Our next result shows that there exists a unique symmetric positive-profits equilibrium provided that segment $A$ 's advertising cost per consumer is low enough compared to segment $B$ 's. To characterize this equilibrium, we exploit the fact that a firm must be indifferent between the distinct pricing and advertising strategies described in Proposition 1.

\footnotetext{
${ }^{14}$ Expression (1) is also useful to understand how our results would be modified if there were economies or diseconomies of scale in advertising. In the presence of strong economies of scale, the entire market would be the most profitable market while in the presence of diseconomies of scale it would be the least profitable market. The result in Proposition 1 would then be modified according to the intuition presented above.
} 
Theorem 2 There exists a unique positive-profits symmetric equilibrium which takes the following form: With probability $\lambda_{A}=\frac{\phi_{B}-\phi_{A} \mu_{B}}{\mu_{B}^{2} R\left(p^{m}\right)+\phi_{B} \mu_{A}}$ firms target their ads on segment $A$ and charge a price $p$ randomly chosen from the set $\sigma_{A}=\left[\underline{p}_{A}, \bar{p}_{A}\right]$ according to the price distribution $F_{A}(p)=$ $1-\frac{\lambda_{B}+\lambda_{M}}{\lambda_{A}} \frac{R\left(\bar{p}_{A}\right)-R(p)}{R(p)}$; with probability $\lambda_{B}=\phi_{A} / \mu_{A} R\left(p^{m}\right)$ firms target their ads on segment $B$ and charge a price $p$ randomly chosen from the set $\sigma_{B}=\left[\underline{p}_{B}, p^{m}\right]$ according to the price distribution $F_{B}(p)=1-\frac{\lambda_{A}-\lambda_{B}}{\lambda_{B}}\left[\frac{R\left(p^{m}\right)-R(p)}{R(p)}\right]$, and with the remaining probability firms advertise in the entire market and charge a price $p$ randomly chosen from the set $\sigma_{M}=\left[\underline{p}_{M}, p^{m}\right]$ according to the price distribution

$$
F_{M}(p)= \begin{cases}1-\frac{\lambda_{A} \mu_{B}\left(R\left(p^{m}\right)-R(p)\right)-\lambda_{B} R(p)+\phi_{A}}{\lambda_{M} R(p)} & \text { for all } p \in\left[\underline{p}_{M}, \underline{p}_{B}\right] \\ 1-\frac{1}{\lambda_{M}}\left(\frac{\phi_{A}}{\mu_{A} R(p)}-\lambda_{B}\right) & \text { for all } p \in\left[\underline{p}_{B}, p^{m}\right]\end{cases}
$$

where $\underline{p}_{A}=R^{-1}\left(\left(1-\lambda_{A}\right) R\left(\bar{p}_{A}\right)\right), \underline{p}_{B}=R^{-1}\left(R\left(p^{m}\right)\left(\lambda_{A}-\lambda_{B}\right) / \lambda_{A}\right)$ and $\bar{p}_{A}=\underline{p}_{M}=R^{-1}\left(\phi_{B} / \mu_{B}\right)$. This equilibrium exists if $\frac{\phi_{A}}{\mu_{A}}<\frac{\phi_{B}}{\mu_{B}}\left(1-\frac{\phi_{B}}{\mu_{B} R\left(p^{m}\right)}\right)$ and firms obtain a profit $E \pi=\mu_{B} \lambda_{A} R\left(p^{m}\right)-$ $\phi_{B}>0$.

The proof is in the Appendix. An interesting feature of the equilibrium described in Theorem 2 is that firms advertise in segment $A$ more frequently than in segment $B$. The reason for this is again related to the inequality (1). Since firms must be indifferent between advertising in either of the segments, in addition to charging lower prices in segment $A$, firms compete for segment $A$ 's consumers more frequently than for segment $B$ 's ones, which makes segment $A$ less attractive in turn. The question that arises is whether pricing is more or less aggressive when firms advertise in the entire market. Our next result provides a response.

Proposition 2 The equilibrium distribution of prices $F_{B}(p)$ dominates $F_{M}(p)$ in a first-order stochastic sense; moreover, from Proposition 1 it follows that $F_{M}(p)$ dominates $F_{A}(p)$ under the same criterion.

The proof is in the Appendix. Since $F_{B}(p)$ dominates $F_{M}(p)$ in a first-order stochastic sense, this implies that expected prices are highest when firms compete for segment $B$ 's buyers, intermediate when they compete for the entire market and lowest when they compete for segment $A$ 's consumers. The reason is that, a priori, entering the entire market is a more attractive strategy than entering 
segment $B$, but a less attractive strategy than entering segment $A$; this pricing behavior helps firms make the distinct entry strategies equally attractive.

The equilibrium described in Theorem 2 is illustrated in Figure 2 below for a situation where the two consumer segments are of equal size and $D(p)=1$ up to the monopoly price $p^{m}=1$. In this graph we have represented the equilibrium distribution of prices advertised in segment $A$, in segment $B$ and in the entire market.

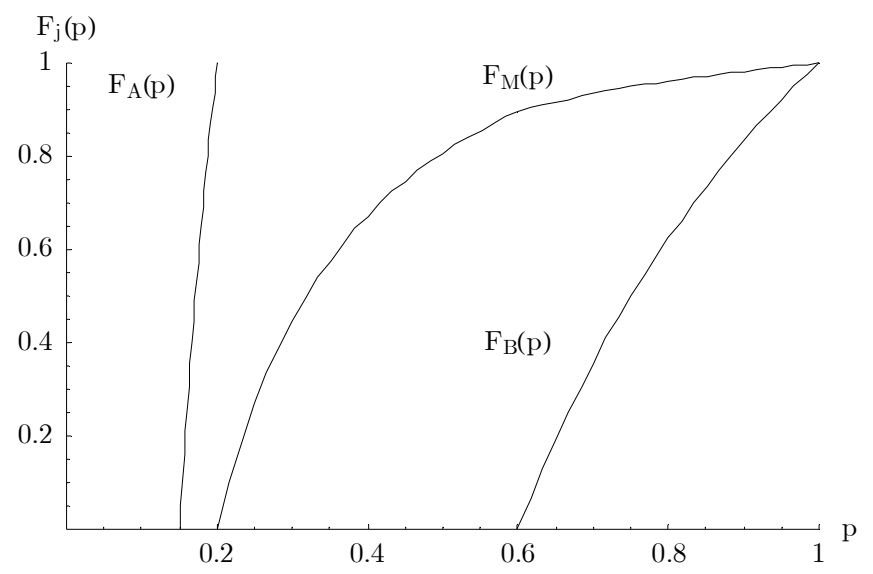

Figure 2: Equilibrium price distributions $\left(\phi_{A}=1 / 20, \phi_{B}=2 / 20\right)$.

We now turn to a discussion of the condition that parameters must satisfy for a positive-profits equilibrium to exist: $\frac{\phi_{A}}{\mu_{A}}<\frac{\phi_{B}}{\mu_{B}}\left(1-\frac{\phi_{B}}{\mu_{B} R\left(p^{m}\right)}\right)$. This condition can easily be represented in the space of per-consumer advertising cost. In Figure 3, a positive-profits equilibrium exists in the region of parameters indicated with the label $E \pi>0$. For any given segment $B$ 's advertising cost, the condition requires segment $A$ 's profitability to be high enough. The reason is as follows. For a given $\phi_{B} / \mu_{B}$, equilibrium profits are equal to $E \pi=\mu_{B} \lambda_{A} R\left(p^{m}\right)-\phi_{B}$, which depend on the probability $\lambda_{A}$ with which a firm does not compete for segment $B$ 's consumers with the rival firm. For this probability to be large enough so that revenues offset the cost of advertising in segment $B$, the profitability of segment $A$ must be sufficiently high. When this is the case, as discussed above, the equilibrium strategy prescribes firms to target their ads on segment $A$ sufficiently frequently. 


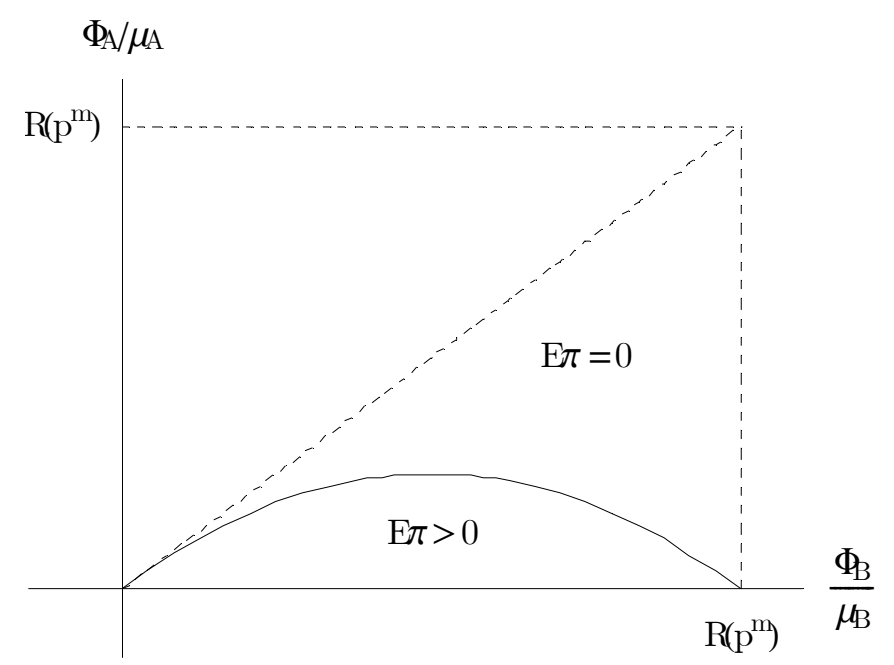

Figure 3: Existence of a positive-profit equilibrium.

The previous discussion motivates an examination of equilibria when parameters violate the condition in Theorem 2. Simple derivations show that $\underline{p}_{M} \rightarrow \underline{p}_{B}, F_{M}(p) \rightarrow F_{B}(p)$ and $E \pi \rightarrow 0$ as $\frac{\phi_{A}}{\mu_{A}} \rightarrow \frac{\phi_{B}}{\mu_{B}}\left(1-\frac{\phi_{B}}{\mu_{B} R\left(p^{m}\right)}\right)$. Moreover, it can be seen that the strategies given in Theorem 2 would yield negative profits whenever $\frac{\phi_{A}}{\mu_{A}}>\frac{\phi_{B}}{\mu_{B}}\left(1-\frac{\phi_{B}}{\mu_{B} R\left(p^{m}\right)}\right)$ because firms do not advertise in segment $A$ sufficiently frequently. We now show that in this case there exists a symmetric equilibrium with zero profits whose characteristics are somewhat similar to those of the positive profits equilibrium of Theorem 2, except in that firms stay out of the market with strictly positive probability. In this equilibrium, the configuration of price supports is as in Figure 4.

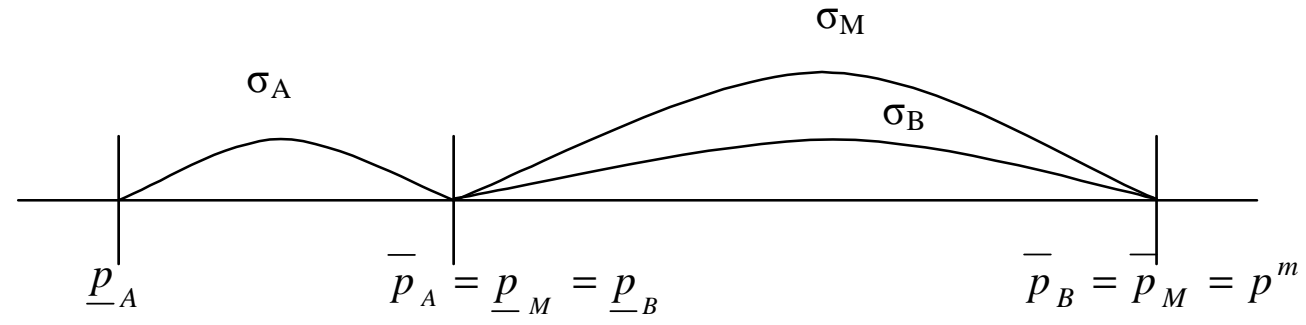

Figure 4: Configuration of price supports in a zero-profits equilibrium.

Theorem 3 Let $\frac{\phi_{A}}{\mu_{A}} \geq \frac{\phi_{B}}{\mu_{B}}\left(1-\frac{\phi_{B}}{\mu_{B} R\left(p^{m}\right)}\right)$. Then there exists a zero-profits symmetric equilibrium which takes the following form: With probability $\lambda_{O}=\frac{\phi_{B}^{2} \mu_{A}-\mu_{B} R\left(p^{m}\right)\left(\phi_{B} \mu_{A}-\phi_{A} \mu_{B}\right)}{\phi_{B} \mu_{B} \mu_{A} R\left(p^{m}\right)}$ firms stay out of the market; with probability $\lambda_{A}=\frac{\phi_{B} \mu_{A}-\phi_{A} \mu_{B}}{\phi_{B} \mu_{A}}$ firms target their ads on segment $A$ and charge a price $p$ randomly chosen from the set $\sigma_{A}=\left[\underline{p}_{A}, \bar{p}_{A}\right]$ according to the price distribution $F_{A}(p)=$ $1-\frac{1}{\lambda_{A}}\left(\frac{\phi_{A}}{R(p) \mu_{A}}-\left(1-\lambda_{A}\right)\right)$; with probability $\lambda_{B}=\lambda_{A}\left(1-\phi_{B} / \mu_{B} R\left(p^{m}\right)\right)$ firms advertise in segment $B$ 
and charge a price $p$ randomly chosen from the set $\sigma_{B}=\left[\underline{p}_{B}, p^{m}\right]$ according to the price distribution $F_{B}(p)=1-\frac{\phi_{B}}{\mu_{B} R\left(p^{m}\right)-\phi_{B}}\left[\frac{R\left(p^{m}\right)-R(p)}{R(p)}\right]$, and with the remaining probability firms advertise in the entire market and charge a price $p$ randomly chosen from the set $\sigma_{M}=\sigma_{B}$ according to the price distribution $F_{M}(p)=F_{B}(p)$, where $\underline{p}_{A}=R^{-1}\left(\phi_{A} / \mu_{A}\right)$, and $\bar{p}_{A}=\underline{p}_{M}=\underline{p}_{B}=R^{-1}\left(\phi_{B} / \mu_{B}\right)$.

This result should be seen as a natural extension of Theorem 2 to a situation where the per consumer advertising cost of the two segments is large enough. As mentioned above, this equilibrium exists precisely in the region of parameters where a positive profits equilibrium fails to exist (region labelled $E \pi=0$ in Figure 3). We note that, for a given segment $B$ 's advertising cost, an increase in segment $A$ 's advertising cost leads to an increase in the probability with which firms stay out of the market. Theorems 2 and 3 together imply that an equilibrium always exists, either with positive profits or with zero-profits. ${ }^{15}$

We now turn to ask how sensitive our equilibria in Theorems 2 and 3 are to parameter changes. The comparative statics results of these two equilibria yield similar insights so we focus in what follows on the positive-profits equilibrium in Theorem 2. For this purpose, let us assume, without loss of generality, that $\phi_{A}=\gamma R\left(p^{m}\right)$, with $\gamma \in\left(0, \mu_{A}\right)$ and $\phi_{B}=\beta \phi_{A}$, with $\beta \in\left[\mu_{B} / \mu_{A}, \mu_{B} / \gamma\right)$. We note that an increase in both segments' advertising fees is captured by an increase in $\gamma$ (keeping constant $\beta$ ) while an increase in the asymmetry across segments is captured either by an increase in $\beta$ or by an increase in $\mu_{A}$.

Proposition 3 In the positive-profits equilibrium described in Theorem 2 the following relations hold:

(1) $\frac{\partial \lambda_{A}}{\partial \gamma}>0, \frac{\partial \lambda_{B}}{\partial \gamma}>0$, and $\frac{\partial \lambda_{M}}{\partial \gamma}<0$. Further, an increase in $\gamma$ widens $\sigma_{A}$ and $\sigma_{B}$, and narrows $\sigma_{M}$. Furthermore, expected profits are non-monotonic in $\gamma$, first increasing and then decreasing. Finally, as $\gamma \rightarrow 0, \lambda_{M} \rightarrow 1$ and $F_{M}(p)$ converges to a price distribution that is degenerate at the marginal cost.

\footnotetext{
${ }^{15}$ For the sake of completeness, we note that other zero-profit symmetric equilibria exist. First, if $\phi_{A} / \mu_{A}=\phi_{B} / \mu_{B}$, then there exist an equilibrium where $\lambda_{O}+\lambda_{M}=1$; we note that this equilibrium is the natural extension of Theorem 1 to the case of segmented markets but only exists if segments are equally attractive. Second, if $\phi_{A} / \mu_{A}=\phi_{B} / \mu_{B}=$ $R\left(p^{m}\right) / 2$, then there exists an equilibium where $\lambda_{A}+\lambda_{B}=1$. Third, if $\phi_{A} / \mu_{A}>R\left(p^{m}\right)-\phi_{B} / \mu_{B}$, then there exists an equilibrium where $\lambda_{O}+\lambda_{A}+\lambda_{B}=1$. Finally, for all parameters, there exists a continuum of equilibria where $\lambda_{O}+\lambda_{A}+\lambda_{B}+\lambda_{M}=1$; this equilibrium differs from that in Theorem 3 in that $\bar{p}_{A}=\bar{p}_{B}=\bar{p}_{M}=p^{m}$. The proofs of these results are available from the authors upon request.
} 
(2) $\frac{\partial \lambda_{A}}{\partial \beta}>0, \frac{\partial \lambda_{B}}{\partial \beta}=0$, and $\frac{\partial \lambda_{M}}{\partial \beta}<0$. Further, an increase in $\beta$ widens $\sigma_{A}$, and narrows both $\sigma_{B}$ and $\sigma_{M}$. Furthermore, expected profits are increasing in $\beta$ if $\beta<2 \mu_{B} / \mu_{A}$; otherwise, expected profits are non-monotonic, first increasing and then decreasing, provided that $\gamma$ is large enough.

(3) $\frac{\partial \lambda_{A}}{\partial \mu_{B}}<0, \frac{\partial \lambda_{B}}{\partial \mu_{B}}>0$, and $\frac{\partial \lambda_{M}}{\partial \mu_{B}}$ is indeterminate. Further, an increase in $\mu_{B}$ narrows $\sigma_{A}$, and widens both $\sigma_{B}$ and $\sigma_{M}$. Furthermore, expected profits are decreasing in $\mu_{B}$ if $\beta<\mu_{B}(1-$ $\left.\mu_{A}\right) / \mu_{A}^{2}$; otherwise expected profits are non-monotonic, first decreasing and then increasing, provided that $\gamma$ is large enough.

Building on this Proposition, we first ask how a decrease in advertising cost $\gamma$ affects the equilibrium. A fall in advertising costs leads to an equilibrium where the market outcome exhibits segmentation less likely and where pricing is less extreme. That is, firms decrease the probability with which they advertise high prices in segment $B$, and also decrease the probability with which they advertise low prices in segment $A$; by implication, firms increase the probability with which they advertise intermediate prices in the entire market. In addition, firms narrow the set of prices they choose from when they enter at the segment level, while they widen the set of prices they choose from when they advertise in the entire market. Keeping everything else constant, what happens is that a decrease in advertising costs makes advertising to all consumers a relatively inexpensive strategy as compared to advertising in just a segment. For firms to remain indifferent between the different advertising strategies, they must decrease competition for the distinct consumer segments and increase competition for the entire market. This results in lower advertising probabilities and lower price dispersion at the segment level, and greater price dispersion at the market level. ${ }^{16}$

How does an increase in advertising costs affect firms' profits? We can show that profits are nonmonotonic in advertising costs, first increasing and then decreasing. We observe that by making advertising in the entire market a less attractive strategy, competitiveness weakens and firm revenues boost. When $\gamma$ is low to begin with, gains from weaker competitiveness offset the advertising cost increase; by contrast, when advertising fees are high enough, the increase in revenues is too

\footnotetext{
${ }^{16}$ We are using here the term price dispersion to refer to the width of the support of a price distribution. This is certainly an abuse of terminology. To be more precise, we have run numerical simulations and found that the variances of the price distributions $F_{A}(p)$ and $F_{B}(p)$ both decrease as $\gamma$ decreases; by contrast we have seen that the variance of $F_{A B}(p)$ exhibits a non-monotonic relationship with respect to $\gamma$, first increasing and then decreasing.
} 
small to outweigh the cost increase. The influence of $\gamma$ on the equilibrium pricing behavior of the firms can be seen in Figure 5. The left graph shows the equilibrium price distributions when advertising costs are low while the right graph represents a market with high advertising costs. We finally notice that as $\gamma$ converges to zero, $\lambda_{M}$ converges to 1 , the equilibrium distribution of prices converges to the degenerate price distribution at the marginal cost and profits converge to zero.
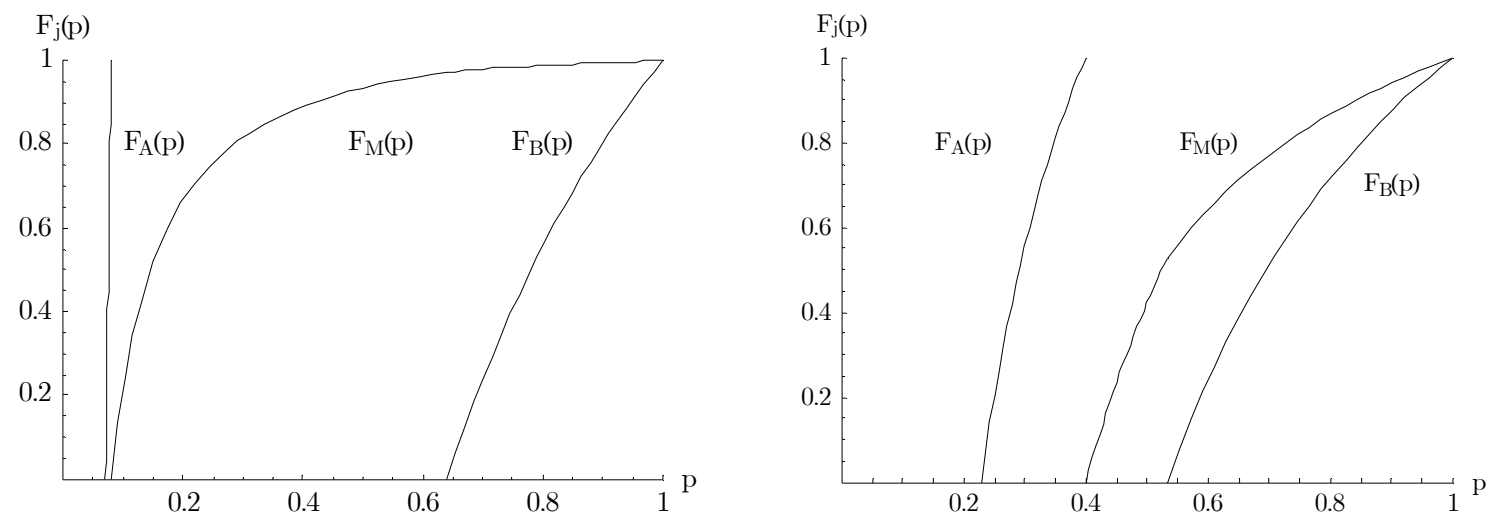

Figure 5a. Low entry $\operatorname{cost}(\gamma=1 / 50 ; \beta=2)$. Figure 5b. High entry cost $(\gamma=1 / 10 ; \beta=2)$.

We now turn to discuss how an increase in the asymmetries across segments influences firm behavior and performance. We start by considering an increase in the difference between the segment $A$ 's advertising cost and segment $B$ 's. An increase in $\beta$ captures an increase in the cost of targeting ads on segment $B$, keeping constant the cost of advertising in segment $A$. Thus, ceteris paribus, an increase in $\beta$ raises asymmetries across segments. This results in an increase in the probability with which firms advertise in segment $A$, while the probability with which firms advertise in segment $B$ is unaltered; as a consequence firms advertise to all the consumers in the market less frequently. The reason is that in equilibrium the three advertising strategies must be equally attractive, so firms must increase competition for segment $A$ 's consumers and decrease competition for segment $B$ 's. The supports of the equilibrium price distributions also change according to intuition (see Figure 6 below). When firms compete more frequently for a segment in the market, they increase price dispersion to mitigate rival's successful price undercutting. ${ }^{17}$ Interestingly, these observations translate into profits being non-monotonic in $\beta$. The reason why profits can increase stems from

\footnotetext{
${ }^{17}$ To be more precise, we note that numerical simulations reveal that the variances of $F_{A}(p)$ and $F_{A B}(p)$ exhibit a non-monotonic relationship with respect to $\beta$, first increasing and then decreasing, while the variance of $F_{B}(p)$ falls in $\beta$.
} 
the weakening of competitiveness associated with the fact that firms compete for the entire market less frequently.

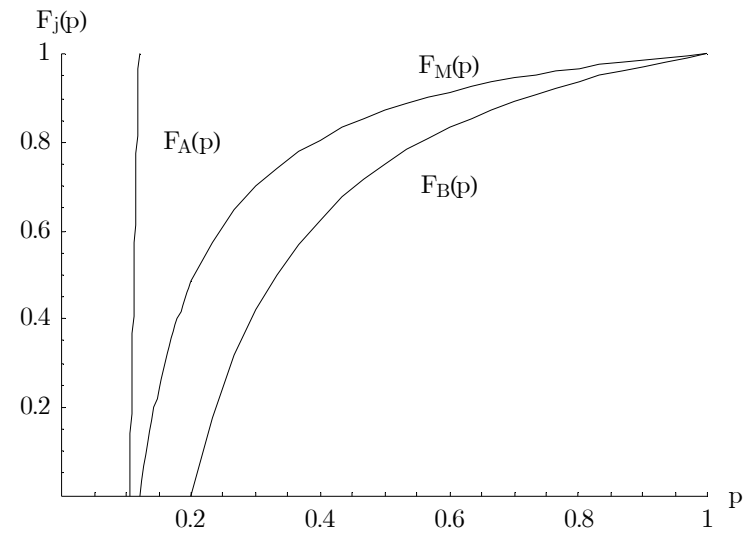

Figure 6a. Low $\beta(\gamma=1 / 20 ; \beta=1.2)$.

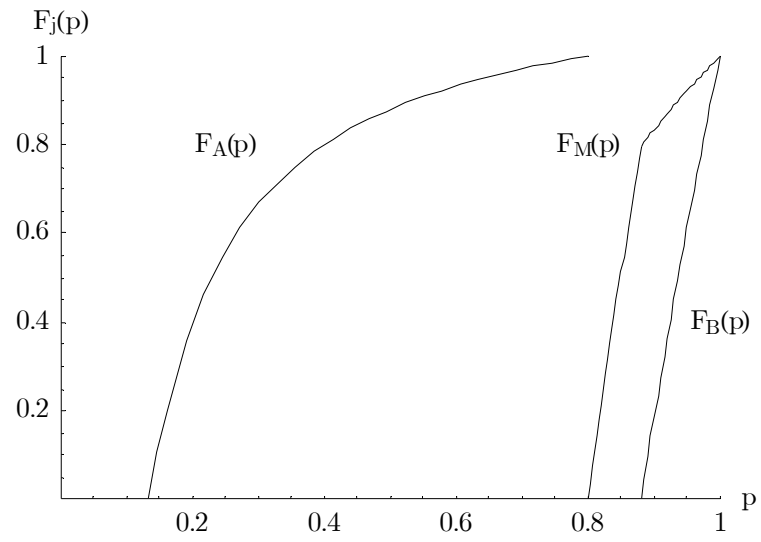

Figure $6 \mathrm{~b}$. High $\beta(\gamma=1 / 20 ; \beta=8)$.

Finally, we discuss the implications of a change in the distribution of consumers across segments. An increase in $\mu_{A}$ leads to an increase in the probability with which firms advertise in segment $A$ and to a decrease in the probability with which they advertise in segment $B$. This results in effects similar to those when $\beta$ rises since this is another way to alter the relative profitability of the two segments. Profits again may exhibit a non-monotonic pattern with respect to the parameter of interest. The reason is as follows. Note that equilibrium profits are $E \pi=\mu_{B} \lambda_{A} R\left(p^{m}\right)-\phi_{B}$. From this expression, it is clear that an increase in $\mu_{A}$ tends to decrease profits, which can be seen as a negative demand effect. However, there is a competition effect by which firms advertise more frequently in segment $A$, which tends to increase profits. We see that, for similar segment sizes, the competition effect is dominant and profits increase as $\mu_{A}$ rises; by contrast, when the sizes of the two segments are already quite different, the demand effect is stronger and profits decrease.

So far we have proved that the use of targeted advertising in segmented markets enables firms to make money in settings where they would only break even in the absence of it; this has been shown for a situation in which segmentation is perfect and two firms operate in the industry. We now investigate the role of these two assumptions. 


\subsection{Imperfect segmentation}

In the market described above, consider now that segmentation is imperfect in the sense that the two consumers segments overlap. This implies that some consumers belong to both segments, i.e., there exists a subset of consumers in segment $A(B)$ who are also in segment $B(A)$. We will denote the total number of consumers who belong to both segments as $\mu_{A B} \cdot{ }^{18}$ Therefore we assume consumer distribution to satisfy $\mu_{A}+\mu_{B}+\mu_{A B}=1$, with $\mu_{i}>0, i=A, B, A B$. We note that, provided that firms do not stay out of the market, the fraction of consumers $\mu_{A B}$ is always fully informed about the prices charged by both firms. Moreover, we note that perfect segmentation obtains as the limiting case $\mu_{A B} \rightarrow 0$.

We start investigating the robustness of the positive-profits equilibrium in Theorem 2. The next result provides a natural extension of Theorem 2 to a market setting in which segmentation is imperfect.

Proposition 4 Let $\frac{\phi_{A}}{\mu_{A}}<\frac{\phi_{B}\left(\mu_{B} R\left(p^{m}\right)-\phi_{B}\right)}{\mu_{B}\left(\mu_{B} R\left(p^{m}\right)-\phi_{B}\right)+\phi_{B}\left(1-\mu_{A}\right)}$. Then, there exists a positive-profits symmetric equilibrium which takes the following form: Let $\underline{p}_{A}=R^{-1}\left(\left(1-\lambda_{A}\right) R\left(\bar{p}_{A}\right)\right), \underline{p}_{M}=\bar{p}_{A}=$ $R^{-1}\left(\frac{\lambda_{A} \mu_{B} R\left(p^{m}\right)+\phi_{A}}{\left(1-\lambda_{A}\right)+\lambda_{A} \mu_{B}}\right)$ and $\underline{p}_{B}=R^{-1}\left(R\left(p^{m}\right) \frac{\lambda_{A} \mu_{B}-\lambda_{B}\left(1-\mu_{A}\right)}{\lambda_{A} \mu_{B}}\right)$. With probability $\lambda_{A}=\frac{\phi_{B}-\phi_{A} \mu_{B}}{\mu_{B}^{2} R\left(p^{m}\right)+\phi_{B}\left(1-\mu_{B}\right)}$ firms target their ads on segment $A$ consumers and charge a price $p$ randomly chosen from the set $\sigma_{A}=\left[\underline{p}_{A}, \bar{p}_{A}\right]$ according to the price distribution $F_{A}(p)=1-\frac{\lambda_{B}+\lambda_{M}}{\lambda_{A}} \frac{R\left(\bar{p}_{A}\right)-R(p)}{R(p)}$; with probability $\lambda_{B}=\frac{\phi_{A}}{\mu_{A} R\left(p^{m}\right)}$ firms advertise a price $p$ randomly chosen from the set $\sigma_{B}=\left[\underline{p}_{B}, p^{m}\right]$ according to the price distribution $F_{B}(p)=1-\frac{\lambda_{A} \mu_{B}-\lambda_{B}\left(1-\mu_{A}\right)}{\left(1-\mu_{A}\right) \lambda_{B}} \frac{R\left(p^{m}\right)-R(p)}{R(p)}$ in segment $B$; and with the remaining probability firms go for the entire market and charge a price $p$ randomly chosen from the set $\sigma_{M}=\left[\underline{p}_{M}, p^{m}\right]$ according to the price distribution

$$
F_{M}(p)= \begin{cases}1-\frac{\lambda_{A} \mu_{B}\left(R\left(p^{m}\right)-R(p)\right)-\lambda_{B} R(p)+\phi_{A}}{\lambda_{M} R(p)} & \text { for all } p \in\left[\underline{p}_{M}, \underline{p}_{B}\right] \\ 1-\frac{1}{\lambda_{M}}\left(\frac{\phi_{A}}{\mu_{A} R(p)}-\lambda_{B}\right) & \text { for all } p \in\left[\underline{p}_{B}, p^{m}\right]\end{cases}
$$

In equilibrium firms obtain profits $E \pi=\lambda_{A} \mu_{B} R\left(p^{m}\right)-\phi_{B}>0$.

\footnotetext{
${ }^{18}$ More formally, consider that $\widetilde{\mu}_{A}$ consumers belong to segment $A$ while $\widetilde{\mu}_{B}$ belong to segment $B$, with $\widetilde{\mu}_{A}+\widetilde{\mu}_{B}=1$. Let $\rho_{A} \widetilde{\mu}_{A}$ be the fraction of segment $A$ consumers who are also part of segment $B$, with $\rho_{A} \in(0,1)$; define similarly $\rho_{B} \widetilde{\mu}_{B}$. Then the total number of consumers who belong to both segments is $\mu_{A B}=\rho_{A} \widetilde{\mu}_{A}+\rho_{B} \widetilde{\mu}_{B}$, while $\mu_{A}=$ $\left(1-\rho_{A}\right) \widetilde{\mu}_{A}$ and $\mu_{B}=\left(1-\rho_{B}\right) \widetilde{\mu}_{B}$.
} 
The proof is in the Appendix. We now discuss the implications of imperfect segmentation on the likelihood of the positive-profits equilibrium described above. We note that the condition in Proposition 4 (imperfect segmentation) converges to the condition in Theorem 2 (perfect segmentation) as $\mu_{A B} \rightarrow 0$. These two conditions are represented in Figure 7 below; the thicker curve represents the condition in Proposition 4 while the thinner curve shows that in Theorem 2. As the size of the fraction of consumers who belong to both segments increases, then the thicker curve shifts downwards, which reduces the set of parameters for which a positive-profits equilibrium exists. This can be seen by comparing the left graph $\left(\mu_{A B}\right.$ small) with the right one ( $\mu_{A B}$ large). The reason for this result is that these consumers increase market competitiveness; indeed, when $\mu_{A B} \rightarrow 1$, then the region under the thicker curve vanishes and firms cannot make money in equilibrium employing the strategy profile given in the Theorem. ${ }^{19}$

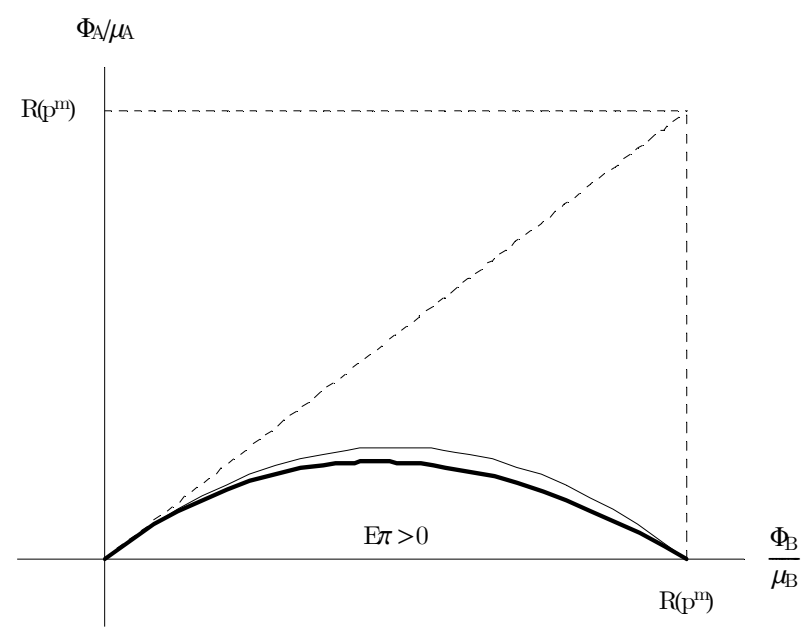

Figure 7a. Low $\mu_{A B}\left(\mu_{A B}=0.15\right)$.

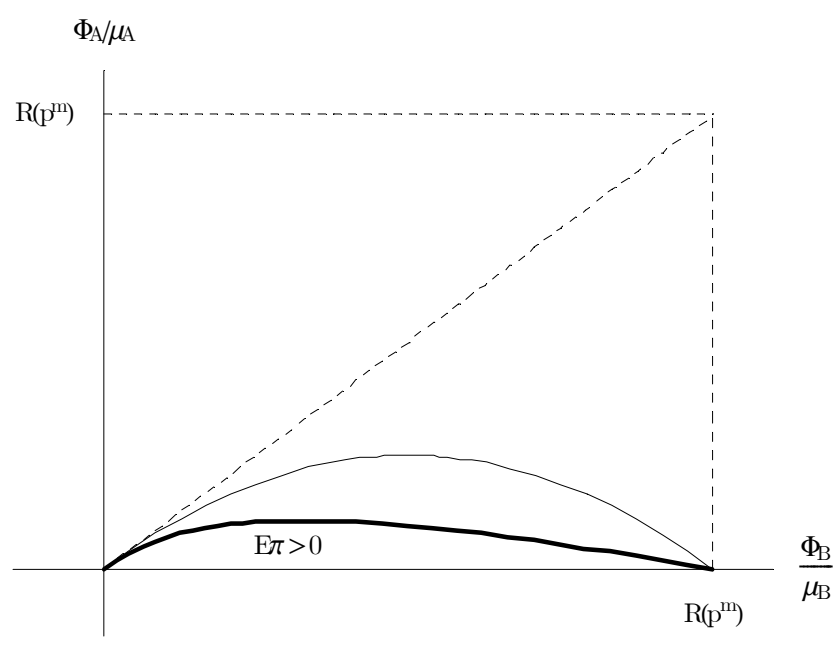

Figure $7 \mathrm{~b}$. High $\mu_{A B}\left(\mu_{A B}=0.4\right)$.

We observe now that imperfect segmentation does not undermine the comparative statics results provided above in the context of Theorem 2 (see Proposition 3). However, a new question arises here: How does an increase in $\mu_{A B}$ affect firm equilibrium behavior and performance? To answer this question, we distinguish between the case in which an increase in $\mu_{A B}$ is accompanied by a decrease in $\mu_{A}$, and the case in which an increase in $\mu_{A B}$ comes with a decrease in $\mu_{B}$.

\footnotetext{
${ }^{19}$ We have proved that there exists another positive-profits equilibrium where firms randomize between advertising low prices in segment $A$ and high prices in segment $B$. This equilibrium exists in a different region of parameters and, interestingly, it covers the entire parameter space as $\mu_{A B} \rightarrow 1$. This implies that imperfect segmentation increases the scope of targeted advertising as a device to soften competition. The proof is available from the authors upon request.
} 
Proposition 5 In the positive-profits equilibrium described above in Proposition 4 the following relations hold:

(1) Holding $\mu_{B}$ constant, $\frac{\partial \lambda_{A}}{\partial \mu_{A B}}=0, \frac{\partial \lambda_{B}}{\partial \mu_{A B}}>0$, and $\frac{\partial \lambda_{M}}{\partial \mu_{A B}}<0$; further, an increase in $\mu_{A B}$ widens $\sigma_{B}$, while $\sigma_{A}, \sigma_{M}$ and equilibrium profits are not altered.

(2) Holding $\mu_{A}$ constant, $\frac{\partial \lambda_{A}}{\partial \mu_{A B}}>0, \frac{\partial \lambda_{B}}{\partial \mu_{A B}}=0$, and $\frac{\partial \lambda_{M}}{\partial \mu_{A B}}<0$; further, an increase in $\mu_{A B}$ widens $\sigma_{A}$ and narrows $\sigma_{M}$; furthermore, if $\gamma<(>) \frac{\mu_{B}^{2}}{\beta-\mu_{B}\left(2-\mu_{B}\right)}$, then, an increase in $\mu_{A B}$ narrows (widens) $\sigma_{B}$ and increases (decreases) equilibrium profit.

The proof is in the Appendix. We now elaborate on the intuition behind this result. When $\mu_{A B}$ increases in such a way that $\mu_{A}+\mu_{A B}$ is constant (part 1), it turns out that segment $A$ remains an equally attractive market (this can be seen by looking at the payoff function (13) in the Appendix). As a result $\lambda_{A}$ does not change. However, segment $B$ becomes a more attractive market; the reason is that as $\mu_{A B}$ rises, the number of customers a firm that advertises in segment $B$ may sell to increases (see equation (14) in the proof). As a result $\lambda_{B}$ increases and $\sigma_{B}$ widens. Finally, an increase in $\mu_{A B}$ makes advertising to all the consumers in the market a less attractive strategy (see equation (15) below). As a result, competition for the entire market must occur less frequently so $\lambda_{M}$ falls to restore equilibrium. Interestingly, profits are insensitive to changes in $\mu_{A B}$ in this case.

When $\mu_{A B}$ increases in such a way that $\mu_{B}+\mu_{A B}$ is constant (part 2 of the Proposition), we note that segment $A$ becomes a more attractive market. The reason is that the number of customers a firm may sell to when advertising in segment $A$ rises (see payoff function (13)). As a result firms must increase competition for segment $A$ buyers and $\sigma_{A}$ correspondingly widens. By contrast, an increase in $\mu_{A B}$ makes advertising in the entire market a less attractive strategy (see (15)) and $\lambda_{M}$ falls to restore the equilibrium. It turns out that equilibrium profits may rise with increasing $\mu_{A B}$; the reason is that firms compete for the entire market less frequently and this reduces overall market competitiveness.

\section{$3.2 \quad$ N-firm oligopoly}

Theorem 2 can be extended to consider an oligopolistic market with $N$ firms. If all firms in the market randomize between targeting their ads on segment $A$ and charging prices from $\sigma_{A}$, sending 
ads to segment $B$ and charging prices from $\sigma_{B}$, and advertising in the entire market and charging prices from $\sigma_{M}$, where $\sigma_{A}, \sigma_{B}$ and $\sigma_{M}$ satisfy Proposition 1, the payoff to a firm advertising $p \in \sigma_{A}$ in segment $A$ is:

$$
\begin{aligned}
& E \pi_{i}\left(\lambda_{A}=1, p \in \sigma_{A} ; s^{-i}\right) \\
& \quad=R(p) \sum_{j=0}^{N-1}\left(\begin{array}{c}
N-1 \\
j
\end{array}\right) \lambda_{A}^{j}\left(1-F_{A}(p)\right)^{j}\left[\sum_{i=0}^{N-1-j}\left(\begin{array}{c}
N-1-j \\
i
\end{array}\right) \lambda_{B}^{i} \lambda_{M}^{N-1-j-i}\right]-\phi_{A}
\end{aligned}
$$

If a firm advertises a price $p$ from $\sigma_{B}$ in segment $B$, this firm obtains a profit equal to:

$$
\begin{aligned}
& E \pi_{i}\left(\lambda_{B}=1, p \in \sigma_{B} ; s^{-i}\right) \\
& =R(p) \sum_{j=0}^{N-1}\left(\begin{array}{c}
N-1 \\
j
\end{array}\right) \lambda_{B}^{j}\left(1-F_{B}(p)\right)^{j}\left[\sum_{i=0}^{N-1-j}\left(\begin{array}{c}
N-1-j \\
i
\end{array}\right) \lambda_{A}^{i} \lambda_{M}^{N-1-j-i}\left(1-F_{M}(p)\right)^{N-1-j-i}\right]-\phi_{B}
\end{aligned}
$$

If a firm advertises in the entire market, it may be the case that this firm charges a price $p \in \sigma_{M} \cap \sigma_{B}$ or else $p \in \sigma_{M} \backslash \sigma_{B}$. The profits a firm obtains in those cases are:

$$
\begin{aligned}
& E \pi_{i}\left(\lambda_{M}=1, p \in \sigma_{M} \cap \sigma_{B} ; s^{-i}\right)=E \pi\left(\lambda_{B}=1, p \in \sigma_{B}\right) \\
& \quad+R(p) \mu_{A}\left[\sum_{j=0}^{N-1}\left(\begin{array}{c}
N-1 \\
j
\end{array}\right) \lambda_{B}^{j} \lambda_{M}^{N-1-j}\left(1-F_{M}(p)\right)^{N-1-j}\right]-\phi_{A}-\phi_{B} \\
& E \pi_{i}\left(\lambda_{M}=1, p \in \sigma_{M} \backslash \sigma_{B} ; s^{-i}\right)=p \mu_{A}\left[\sum_{j=0}^{N-1}\left(\begin{array}{c}
N-1 \\
j
\end{array}\right) \lambda_{B}^{j} \lambda_{M}^{N-1-j}\left(1-F_{M}(p)\right)^{N-1-j}\right] \\
& \quad+R(p) \mu_{B} \sum_{j=0}^{N-1}\left(\begin{array}{c}
N-1 \\
j
\end{array}\right) \lambda_{A}^{j}\left[\sum_{i=0}^{N-1-j}\left(\begin{array}{c}
N-1-j \\
i
\end{array}\right) \lambda_{B}^{i} \lambda_{M}^{N-1-j-i}\left(1-F_{M}(p)\right)^{N-1-j-i}\right]-\phi_{A}-\phi_{B} .
\end{aligned}
$$

Using the binomial theorem, these profit expressions can be simplified:

$$
\begin{aligned}
E \pi_{i}\left(\lambda_{A}=\right. & \left.1, p \in \sigma_{A} ; s^{-i}\right)=R(p) \mu_{A}\left[1-\lambda_{A}+\lambda_{A}\left(1-F_{A}(p)\right]^{N-1}-\phi_{A}\right. \\
E \pi_{i}\left(\lambda_{B}=\right. & \left.1, p \in \sigma_{B} ; s^{-i}\right)=R(p) \mu_{B}\left[\lambda_{A}+\lambda_{M}\left(1-F_{M}(p)\right)+\lambda_{B}\left(1-F_{B}(p)\right)\right]^{N-1}-\phi_{B} \\
E \pi_{i}\left(\lambda_{M}=\right. & \left.1, p \in \sigma_{M} \cap \sigma_{B} ; s^{-i}\right)=E \pi\left(\lambda_{B}=1, p \in \sigma_{B}\right)+R(p) \mu_{A}\left[\lambda_{B}+\lambda_{M}\left(1-F_{M}(p)\right)\right]^{N-1}-\phi_{A}-\phi_{B} \\
E \pi_{i}\left(\lambda_{M}=\right. & \left.1, p \in \sigma_{M} \backslash \sigma_{B} ; s^{-i}\right)=R(p) \mu_{A}\left[\lambda_{B}+\lambda_{M}\left(1-F_{M}(p)\right)\right]^{N-1} \\
& +R(p) \mu_{B}\left[\lambda_{A}+\lambda_{B} \lambda_{M}\left(1-F_{M}(p)\right)\right]^{N-1}-\phi_{A}-\phi_{B} .
\end{aligned}
$$

A similar procedure as the one that underlies Theorem 2 enables us to find a characterization of equilibrium in this $N$-firm market. Our interest is on how entry of firms affects equilibrium profits. 
The profits a firm obtains from advertising a price $\underline{p}_{M}$ to all the consumers in the market must be equal to the profits this firm gets if it charges $p^{m}$ instead. This yields an expression for the equilibrium frequency with which firms target their ads on segment $A$ :

$$
R\left(p^{m}\right) \mu_{B} \lambda_{A}^{N-1}-\frac{\mu_{A}}{\mu_{B}} \phi_{B}\left(1-\lambda_{A}\right)^{N-1}-\phi_{B}+\phi_{A}=0
$$

Unfortunately this expression cannot be solved for $\lambda_{A}$ explicitly. However we note that equilibrium profits are given by

$$
E \pi=R\left(p^{m}\right) \mu_{B} \lambda_{A}^{N-1}-\phi_{B}
$$

so, using (2), we can examine how profits change with the number of firms:

$$
\begin{aligned}
\frac{d \lambda_{A}^{N-1}}{d N} & =(N-1) \frac{\partial \lambda_{A}}{\partial N}+\lambda_{A} \ln \left[\lambda_{A}\right] \\
& =(N-1) \frac{-R\left(p^{m}\right) \mu_{B} \lambda_{A}^{N-1} \ln \left[\lambda_{A}\right]+\frac{\mu_{A}}{\mu_{B}} \phi_{B}\left(1-\lambda_{A}\right)^{N-1} \ln \left[1-\lambda_{A}\right]}{R\left(p^{m}\right) \mu_{B}(N-1) \lambda_{A}^{N-2}+\frac{\mu_{A}}{\mu_{B}} \phi_{B}(N-1)\left(1-\lambda_{A}\right)^{N-2}}+\lambda_{A} \ln \left[\lambda_{A}\right] \\
& =\frac{\frac{\mu_{A}}{\mu_{B}} \phi_{B}\left(1-\lambda_{A}\right)^{N-1} \ln \left[1-\lambda_{A}\right]+\frac{\mu_{A}}{\mu_{B}} \phi_{B}\left(1-\lambda_{A}\right)^{N-2} \lambda_{A} \ln \left[\lambda_{A}\right]}{R\left(p^{m}\right) \mu_{B} \lambda_{A}^{N-2}+\frac{\mu_{A}}{\mu_{B}} \phi_{B}\left(1-\lambda_{A}\right)^{N-2}}<0 .
\end{aligned}
$$

As a result:

Proposition 6 Consider an $N$-firms oligopoly advertising and pricing game where $\mu_{A}+\mu_{B}=$ $1, \mu_{j}>0, j=A, B$. Assume firms strategies $s^{i}=\left\{\left(\lambda_{j}^{i}, F_{j}^{i}(p)\right)\right\}_{j \in E_{i}, i=1, \ldots, N}$ satisfy Proposition 1. Then, a positive-profits equilibrium exists and entry of firms causes equilibrium profits to fall.

\section{Price discrimination}

In Section 3, a firm deciding to send ads to all the consumers in the market did charge a single price. As argued above (see footnote 11) this is a reasonable assumption in many settings. However, there may be situations where firms find it feasible to print advertisements with different prices and send them to distinct consumer segments at the same time. This motivates an examination of the implications of allowing firms to price discriminate across segments. Compared to the benchmark case of no segmentation, our next result shows that targeted advertising brings about different types of equilibria but all equilibria exhibit zero-profits under price discrimination. 
Proposition 7 Let $\mu_{A}+\mu_{B}=1, \mu_{j}>0, j=A, B$. Consider the advertising and pricing game described above; if firms can practise price discrimination when advertising in the entire market, then firms obtain zero-profits in equilibrium. The following strategies constitute an equilibrium for all parameters: firms stay out of the market with probability $\lambda_{O}=\frac{\phi_{A}+\phi_{B}}{R\left(p^{m}\right)}$ and enter the entire market with probability $\lambda_{M}=1-\lambda_{O}$ in which case they charge segment $j$ 's consumers a price $p$ randomly chosen from the set $\sigma_{j}=\left[R^{-1}\left(\phi_{j} / \mu_{j}\right), p^{m}\right]$ according to the price distribution $F_{j}(p)=$ $1-\frac{1}{\lambda_{M}}\left(\frac{\phi_{j}}{\mu_{j} R(p)}-\lambda_{O}\right), j=A, B$. There are other zero-profits equilibria: in particular, there exists an equilibrium where $\lambda_{O}+\lambda_{A}+\lambda_{B}=1$ and a continuum of equilibria where $\lambda_{O}+\lambda_{A}+\lambda_{B}+\lambda_{M}=1$.

The proof, which is in the Appendix, proceeds as follows. We first note that pure-strategy equilibria do not exist; second, we show that whenever strategies prescribe a firm to advertise a price $p_{A}$ in segment $A$ and a price $p_{B}$ in segment $B$, then such a firm finds it profitable to deviate by advertising a pair of prices $\left(p_{A}, p_{B}\right)$ in the entire market. The fact that a firm can deviate and charge distinct prices in different consumer segments is what distinguishes this result from that in Theorem 2 .

This result constitutes another instance showing that firms may benefit from bans on price discrimination. Other cases have been put forward by Holmes (1989) and Thisse and Vives (1988). The intuition behind the fact that firms cannot obtain positive profits when they price discriminate across segments is that such pricing policy allows sellers to treat the distinct consumer segments as completely separate markets. In such a case, a firm's problem is separable in markets and, for example, a natural extension of Theorem 1 can be supported as symmetric equilibrium.

\section{Conclusions}

We have examined a strategic game of targeted advertising and pricing between sellers of homogeneous products that operate in a market with various consumer segments. We have seen that if the market were not segmented, or if firms were unable to target their ads on particular consumer groups, competition would drive firm profits down to zero; by contrast, when firms can use targeted advertising and the distinct market segments differ sufficiently in terms of their profitability, firms obtain positive profits in equilibrium. This has illustrated the idea that segmentation mitigates market competitiveness provided that firms have advertising technologies that allow for the targeting of ads at their disposal. Firms equilibrium strategies involve random pricing and random 
advertising and have the property that firm aggressiveness in a given market segment is inversely related to the relative profitability of the segment in question. As a result, firms advertise low prices in the most profitable segment, high prices in the least profitable segment and intermediate prices in the entire market. This implies that neither high nor low prices are heavily advertised but intermediate ones.

These results have been derived in a model where consumer segmentation is 'perfect' in the sense that the distinct buyer segments do not overlap; however, the features of this equilibrium prove to be robust to 'imperfect' consumer segmentation. More interestingly, we have seen that firms may obtain higher profits when segmentation becomes more imperfect. We have also explored the implications of entry of firms and the analysis has revealed that firms' profits fall as the market hosts more sellers. Finally, we have examined the role that price discrimination would have in our model and we have found that firms would benefit from restrictions on price discrimination when they engage in advertising and pricing competition in segmented markets.

All these results have been obtained in a model where firms sell homogeneous products, and, to make it tractable, where advertising reaches all consumers and buyers do not search. However, the idea that targeted advertising may weaken market competitiveness should easily extend to the case of imperfect advertising technologies, consumer search, and product differentiation.

The results of this paper have been derived in a market with only two consumers segments. Certainly, markets are often comprised by a larger number of consumer segments, say $m$. A fascinating question is then whether a market with $m+1$ consumer segments is more or less competitive than a market with $m$ segments. Unfortunately, the game examined above becomes non-tractable when there are $m$ segments in the consumer population so we have been unable to answer this question in general. The problem is that there are $2^{m+1}-1$ possible advertising strategies that must be considered. Perhaps numerical methods can help tackle this problem in general; this is left open for further research. 


\section{Appendix}

Proof of Theorem 1: It is easy to see that pure advertising-strategy profiles cannot be part of an equilibrium. As a consequence, firms must randomize between staying out of the market and advertising in the entire market, i.e., $\lambda_{O}+\lambda_{M}=1, \lambda_{j} \in(0,1), j=O, M$. Let us denote firm $i$ 's strategy as $s^{i}=\left\{\lambda_{O},\left(\lambda_{M}, F_{M}(p)\right)\right\}, i=1,2$. It is easy to check that $E \pi_{i}\left(\lambda_{M}=1, p ; s^{-i}\right)=$ $R(p)\left[\lambda_{O}+\lambda_{M}\left(1-F_{M}(p)\right)\right]-\phi_{A}-\phi_{B}=0$ for any $p \in \sigma_{M}$ only if $\lambda_{O}, \lambda_{M}, F_{M}(p)$ and $\sigma_{M}$ take the form specified in Theorem $1, i=1,2$. Moreover, it is readily seen that firms do not have an incentive to deviate, that $\lambda_{j} \in(0,1), j=O, M$ and that $F_{M}(p)$ is a well-behaved distribution function defined over the set $\sigma_{M}$ for any $\phi_{j}, \mu_{j}, j=A, B$. This completes the proof.

Proof of Lemma 1: It is obvious that any strategy profile in which a firm (or both) stays out of the market with probability one is not part of an equilibrium. We then concentrate on advertising-strategy profiles where firms advertise in some market with strictly positive probability. We start with symmetric pure strategy profiles. Suppose that both firms advertise in segment $j$ with probability one, $j=A, B, M$. If this were an equilibrium firms would advertise a price equal to marginal cost; but in this case firms would not cover their advertising costs. Thus, this cannot be part of an equilibrium.

We now rule out asymmetric pure advertising-strategy profiles. Suppose firms advertise in different segments, e.g., firm 1 advertises in segment $A$ while firm 2 advertises in segment $B$. Then, firms should charge $p^{m}$, which would yield profits $\pi_{1}=\mu_{A} R\left(p^{m}\right)-\phi_{A}$ and $\pi_{2}=\mu_{B} R\left(p^{m}\right)-\phi_{B}$ to the firms. But if this were an equilibrium, a firm would find it profitable to deviate by advertising a price slightly lower than the rival's in its segment . Finally, assume that a firm, say firm 1, advertises just in a single segment, say $A$, and the other firm goes for the entire market. Let $s^{1}=\left\{\lambda_{A}=1, F_{A}(p)\right\}$ be firm 1's strategy and let $s^{2}=\left\{\lambda_{M}=1, F_{M}(p)\right\}$ denote firm 2's strategy. If this were an equilibrium firms' profits would be given by:

$$
\begin{aligned}
& E \pi_{1}\left(\lambda_{A}=1, p ; s^{2}\right)=R(p) \mu_{A}\left(1-F_{M}(p)\right)-\phi_{A} \\
& E \pi_{2}\left(\lambda_{M}=1, p ; s^{1}\right)=R(p)\left[\mu_{A}\left(1-F_{A}(p)\right)+\mu_{B}\right]-\phi_{A}-\phi_{B} .
\end{aligned}
$$

We note that it must be the case that $\bar{p}_{A}<\bar{p}_{M}$ because otherwise firm 1 advertising the upper 
bound $\bar{p}_{A}$ in $A$ would make negative profits. Since firm 2's profits must be constant for all prices in $\sigma_{M}$, it follows that firm 2's expected profits must be $E \pi_{2}=\mu_{B} R\left(\bar{p}_{M}\right)-\phi_{A}-\phi_{B}$. Now it is obvious that firm 2 would gain by exiting segment $A$ since the firm would save on advertising costs. The other pure entry-strategy profiles are ruled out similarly. The proof is now complete.

Proof of Proposition 1: (a) We first show that a symmetric positive-profits equilibrium exists only if $\lambda_{j} \in(0,1) j=A, B, M$. The result is proved by contradiction. Lemma 1 implies that an equilibrium with positive profits exists only if firms employ a random advertising-strategy. It is obvious that any strategy profile prescribing a firm to stay out of the market with positive probability cannot yield positive profits in equilibrium. Thus, we concentrate on mixed advertisingstrategy profiles where $\lambda_{O}=0$. Suppose, first, that firms randomize between advertising in segment $A$ and advertising in segment $B$, i.e., $\lambda_{A}+\lambda_{B}=1, \lambda_{j}>0, j=A, B$. Let us denote firms' strategies as $s^{i}=\left\{\left(\lambda_{A}, F_{A}(p)\right),\left(\lambda_{B}, F_{B}(p)\right)\right\}, i=1,2$. Given the rival's strategy, firm $i$ 's payoff from advertising a price $p$ in segment $A$ is

$$
E \pi_{i}\left(\lambda_{A}=1, p ; s^{-i}\right)=R(p) \mu_{A}\left[\lambda_{A}\left(1-F_{A}(p)\right)+\lambda_{B}\right]-\phi_{A} .
$$

It is easy to see that the upper bound of $F_{A}(p)$ cannot be lower than $p^{m}$ because otherwise a firm advertising the upper bound in $A$ would gain by slightly raising its price, i.e., $\bar{p}_{A}=p^{m}$. For analogous reasons, it must be the case that $\bar{p}_{B}=p^{m}$. This implies that if this were an equilibrium then $E \pi_{i}\left(\lambda_{A}=1, p^{m} ; s^{-i}\right)=E \pi_{i}\left(\lambda_{B}=1, p^{m} ; s^{-i}\right)>0$. But then a firm would gain by deviating and advertising $p^{m}$ in the entire market; indeed, deviating profits would be $E \pi_{i}^{d}\left(\lambda_{M}=1, p^{m} ; s^{-i}\right)=$ $E \pi_{i}\left(\lambda_{B}=1, p^{m} ; s^{-i}\right)+E \pi_{i}\left(\lambda_{A}=1, p^{m} ; s^{-i}\right)$.

Second, suppose that $\lambda_{A}+\lambda_{M}=1, \lambda_{j}>0, j=A, M$. Let's denote firm $i$ 's strategy as $s^{i}=$ $\left\{\left(\lambda_{A}, F_{A}(p)\right),\left(\lambda_{M}, F_{M}(p)\right)\right\}, i=1,2$. Taking as given the rival's strategy, the profit to firm $i$ from advertising $p$ in segment $A$ is

$$
E \pi_{i}\left(\lambda_{A}=1, p ; s^{-i}\right)=R(p) \mu_{A}\left[\lambda_{A}\left(1-F_{A}(p)\right)+\lambda_{M}\left(1-F_{M}(p)\right)\right]-\phi_{A} .
$$


Likewise, the profit to firm $i$ from advertising $p$ in the entire market is

$$
E \pi_{i}\left(\lambda_{M}=1, p ; s^{-i}\right)=\lambda_{A} R(p) \mu_{B}+\lambda_{A} \mu_{A} R(p)\left(1-F_{A}(p)\right)+\lambda_{M} R(p)\left(1-F_{M}(p)\right)-\phi_{A}-\phi_{B} .
$$

As above we observe that $F_{A}(p)$ and $F_{M}(p)$ cannot have atoms. We now note that $\bar{p}_{A}<\bar{p}_{M}$; indeed if $\bar{p}_{A} \geq \bar{p}_{M}$ a firm setting the upper bound $\bar{p}_{A}$ in segment $A$ would always obtain negative profits. Observe next that it must be the case that $\bar{p}_{M}=p^{m}$; this is because there is a strictly positive probability that a firm that advertises in the entire market is the only firm that is active in segment $B$; then, a firm advertising a different upper bound would gain by raising its price. Firms must be indifferent between the distinct price and advertising strategies; therefore equilibrium profits would be: $E \pi_{i}\left(\lambda_{M}=1, p^{m} ; s^{-i}\right)=\lambda_{A} R\left(p^{m}\right) \mu_{B}-\phi_{A}-\phi_{B}$. We now note that a firm can gain by advertising $p^{m}$ only in segment $B$. Indeed, profits to firm $i$ from such a deviation are $E \pi_{i}^{d}\left(\lambda_{B}=1, p^{m} ; s^{-i}\right)=\lambda_{A} R\left(p^{m}\right) \mu_{B}-\phi_{B}$, which are clearly greater than equilibrium profits for all $\phi_{A}>0$. The case in which $\lambda_{B}+\lambda_{M}=1, \lambda_{j} \in(0,1) j=B, M$ is ruled out similarly.

(b) We now prove that a symmetric positive-profits equilibrium exists only if $F_{A}(p), F_{B}(p)$ and $F_{M}(p)$ are atomless. Let $s^{i}=\left\{\left(\lambda_{A}, F_{A}(p)\right),\left(\lambda_{B}, F_{B}(p)\right),\left(\lambda_{M}, F_{M}(p)\right)\right\}, i=1,2$ denote firms' strategies. The expected payoff to a firm from the different entry actions is:

$$
\begin{aligned}
E \pi_{i}\left(\lambda_{A}=1, p ; s^{-i}\right) & =p \mu_{A}\left[\lambda_{A}\left(1-F_{A}(p)\right)+\lambda_{B}+\lambda_{M}\left(1-F_{M}(p)\right)\right]-\phi_{A} \\
E \pi_{i}\left(\lambda_{B}=1, p ; s^{-i}\right) & =p \mu_{B}\left[\lambda_{A}+\lambda_{B}\left(1-F_{B}(p)\right)+\lambda_{M}\left(1-F_{M}(p)\right)\right]-\phi_{B} \\
E \pi_{i}\left(\lambda_{M}=1, p ; s^{-i}\right) & =E \pi_{i}\left(\lambda_{A}=1, p ; s^{-i}\right)+E \pi_{i}\left(\lambda_{B}=1, p ; s^{-i}\right)
\end{aligned}
$$

By inspection of the profits expression above it is easy to see that atoms can be profitably undercut. Hence, the proof follows.

(c) Finally, we prove that a symmetric equilibrium exists only if $\underline{p}_{A}<\underline{p}_{M}=\bar{p}_{A}<\underline{p}_{B}<\bar{p}_{M}=\bar{p}_{B}=$ $p^{m}$. The proof follows from a series of seven claims.

Claim 1: If a positive-profits equilibrium exists, $\nexists p \in \sigma_{A} \cap \sigma_{B}$.

Proof. We show this by contradiction. Suppose there existed a price common to $\sigma_{A}$ and $\sigma_{B}$. Then if a firm were indifferent between advertising such a price in segment $A$ and in segment $B$, and 
that price yielded positive profits, the firm would gain by deviating and advertising it in the entire market, which yields a contradiction.

Claim 2: If a positive-profits equilibrium exists, $\underline{p}_{A}<\underline{p}_{B}$.

Proof. Suppose on the contrary that $\underline{p}_{A}>\underline{p}_{B}$ (the case $\underline{p}_{A}=\underline{p}_{B}$ is ruled out by Claim 1). In equilibrium the expected profit to a firm advertising $\underline{p}_{B}$ in segment $B$ is $E \pi_{i}\left(\lambda_{B}=1, \underline{p}_{B} ; s^{-i}\right)=$ $R\left(\underline{p}_{B}\right) \mu_{B}\left[\lambda_{A}+\lambda_{B}+\lambda_{M}\left(1-F_{M}\left(\underline{p}_{B}\right)\right)\right]-\phi_{B}$. However note that if a firm advertises the same price $\underline{p}_{B}$ in segment $A$ the expected profit is $E \pi_{i}\left(\lambda_{A}=1, \underline{p}_{B} ; s^{-i}\right)=R\left(\underline{p}_{B}\right) \mu_{A}\left[\lambda_{A}+\lambda_{B}+\lambda_{M}\left(1-F_{M}\left(\underline{p}_{B}\right)\right)\right]-\phi_{A}$. Since $\phi_{A} / \mu_{A} \leq \phi_{B} / \mu_{B}$ then $E \pi_{i}\left(\lambda_{A}=1, \underline{p}_{B} ; s^{-i}\right) \geq E \pi_{i}\left(\lambda_{B}=1, \underline{p}_{B} ; s^{-i}\right)>0$. As a result, a firm setting $\underline{p}_{B}$ in segment $B$ would gain by deviating and advertising $\underline{p}_{B}$ in the entire market.

Claim 3: If a positive-profits equilibrium exists, $\underline{p}_{M} \leq \bar{p}_{A}$.

Proof. Suppose not and $\underline{p}_{M}>\bar{p}_{A}$. Then a firm advertising $\bar{p}_{A}$ in segment $A$ would gain by slightly increasing its price.

Claim 4: If a positive-profits equilibrium exists, $\bar{p}_{M}>\underline{p}_{B}$.

Proof. Suppose, on the contrary, that $\bar{p}_{M} \leq \underline{p}_{B}$. Consider first that $\bar{p}_{M}<\underline{p}_{B}$. We have two possibilities. One, $\bar{p}_{A} \leq \bar{p}_{M}<\underline{p}_{B}$; in this case, a firm advertising $\bar{p}_{M}$ in the entire market would strictly gain by increasing the price until $\underline{p}_{B}$. Two, $\bar{p}_{M}<\bar{p}_{A}<\underline{p}_{B}$; if this is the case then a firm advertising $\bar{p}_{A}$ in $A$ would gain by raising its price until $p^{m}$, which contradicts Claim 1 . It remains to prove that $\bar{p}_{M}=\underline{p}_{B}$ cannot be part of an equilibrium. We start noting that it must be the case that $\bar{p}_{M}=\underline{p}_{B}>\bar{p}_{A}$. To see this note that if $\bar{p}_{M}=\underline{p}_{B} \leq \bar{p}_{A}$ then a firm advertising $\bar{p}_{A}$ in segment $A$ would gain by raising its price. Further, given $\bar{p}_{M}=\underline{p}_{B}>\bar{p}_{A}$ it follows that $\bar{p}_{B}=p^{m}$ and therefore firms' expected profit in equilibrium is $\lambda_{A} \mu_{B} R\left(p^{m}\right)-\phi_{B}>0$. Furthermore, in equilibrium $E \pi_{i}\left(\lambda_{M}=1, \bar{p}_{M} ; s^{-i}\right)=E \pi_{i}\left(\lambda_{B}=1, \bar{p}_{M} ; s^{-i}\right)$, which is satisfied if and only if $\lambda_{B} \mu_{A} R\left(\bar{p}_{M}\right)-\phi_{A}=0$. However we note that if a firm deviates by advertising $p=p^{m}>\bar{p}_{M}$ in the entire market, this firm will obtain profits equal to $E \pi_{i}^{d}\left(\lambda_{M}=1, p^{m} ; s^{-i}\right)=\lambda_{A} \mu_{B} R\left(p^{m}\right)-\phi_{B}+$ $\lambda_{B} \mu_{A} R\left(p^{m}\right)-\phi_{A}>\lambda_{A} \mu_{B} R\left(p^{m}\right)-\phi_{B}$.

Claim 5: If a positive-profits equilibrium exists, $\bar{p}_{M}=\bar{p}_{B}=p^{m}$.

Proof. We prove this by contradiction. First, suppose that $\bar{p}_{B}<\bar{p}_{M}$. The support configuration would then be $\underline{p}_{M} \leq \bar{p}_{A}<\underline{p}_{B}<\bar{p}_{B}<\bar{p}_{M}=p^{m}$, where $\bar{p}_{M}=p^{m}$ because otherwise a firm entering 
the entire market and charging $\bar{p}_{M}$ would gain by increasing its price. A firm must be indifferent between charging any price $p \in\left[\bar{p}_{B}, p^{m}\right]$ in the entire market. Then, it must be the case that $E \pi_{i}\left(\lambda_{M}=1, p ; s^{-i}\right)=E \pi_{i}\left(\lambda_{M}=1, p^{m} ; s^{-i}\right)$, which, using (5), yields:

$$
F_{M}(p)=1-\frac{\lambda_{A} \mu_{B}+\lambda_{B} \mu_{A}}{\lambda_{M}}\left(\frac{R\left(p^{m}\right)-R(p)}{R(p)}\right), \text { for all } p \in\left[\bar{p}_{B}, p^{m}\right]
$$

Moreover, a firm must be indifferent between charging any price $p \in\left[\underline{p}_{B}, \bar{p}_{B}\right]$ in segment $B$ or in the entire market. Thus, it must hold that $E \pi_{i}\left(\lambda_{M}=1, p ; s^{-i}\right)=E \pi_{i}\left(\lambda_{B}=1, p ; s^{-i}\right)$, which, using (4) and (5), yields:

$$
F_{M}(p)=1-\frac{1}{\lambda_{M}}\left(\frac{\phi_{A}}{\mu_{A} R(p)}-\lambda_{B}\right) \text { for all } p \in\left[\underline{p}_{B}, \bar{p}_{B}\right]
$$

The price distributions (6) and (7) must be equal at $p=\bar{p}_{B}$. Imposing this condition we obtain:

$$
R\left(\bar{p}_{B}\right)=\frac{\left(\lambda_{A} \mu_{B}+\lambda_{B} \mu_{A}\right) R\left(p^{m}\right)-\frac{\phi_{A}}{\mu_{A}}}{\left(\lambda_{A}-\lambda_{B}\right) \mu_{B}}
$$

A firm must be indifferent between entering segment $B$ and setting a price $p \in \sigma_{B}$ and entering the entire market and charging $p^{m}$, i.e., $E \pi_{i}\left(\lambda_{B}=1, p ; s^{-i}\right)=E \pi_{i}\left(\lambda_{M}=1, p^{m} ; s^{-i}\right)$. Using (7), this yields an expression for the distribution of prices charged in segment $B$ :

$$
F_{B}(p)=\frac{\phi_{A}}{\mu_{A} \mu_{B} \lambda_{B} R(p)}+\frac{\lambda_{A}}{\lambda_{B}}-\frac{\lambda_{A} \mu_{B}+\lambda_{B} \mu_{A}}{\lambda_{B} \mu_{B}} \frac{R\left(p^{m}\right)}{R(p)}
$$

We can now determine the lowerbound of $\sigma_{B}$ by solving $F_{B}\left(\underline{p}_{B}\right)=0$ in (8):

$$
R\left(\underline{p}_{B}\right)=\frac{\left(\lambda_{A} \mu_{B}+\lambda_{B} \mu_{A}\right) R\left(p^{m}\right)-\frac{\phi_{A}}{\mu_{A}}}{\lambda_{A} \mu_{B}}
$$

Since $\underline{p}_{B}$ must be positive in equilibrium, it must be the case that $\left(\lambda_{A} \mu_{B}+\lambda_{B} \mu_{A}\right) R\left(p^{m}\right)-\frac{\phi_{A}}{\mu_{A}}>0$. Since $\bar{p}_{B}$ must also be positive, this implies that $\lambda_{A}-\lambda_{B}>0$. Now we can compare $\bar{p}_{B}$ and $p^{m}$. For $\bar{p}_{B}<p^{m}$ to hold, it must be the case that $\lambda_{B} \mu_{A} R\left(p^{m}\right)-\phi_{A}<0$; but this implies that a firm charging $p^{m}$ in the entire market, which makes a profit equal to $\lambda_{A} \mu_{B} R\left(p^{m}\right)-\lambda_{B} \mu_{A} R\left(p^{m}\right)-\phi_{A}-\phi_{B}$, would gain by entering only segment $B$. This constitutes a contradiction and proves that $\bar{p}_{B} \geq \bar{p}_{M}$. 
It remains to prove that $\bar{p}_{B}>\bar{p}_{M}$ cannot be part of an equilibrium. If this were so, then $\bar{p}_{B}=p^{m}$, and therefore the support configuration would be $\underline{p}_{M} \leq \bar{p}_{A}<\underline{p}_{B}<\bar{p}_{M}<\bar{p}_{B}=p^{m}$. Moreover, in equilibrium $E \pi_{i}\left(\lambda_{B}=1, p^{m} ; s^{-i}\right)=\lambda_{A} \mu_{B} R\left(p^{m}\right)-\phi_{B}$. We know that a firm which deviates by entering the entire market and charging $p^{m}$ would obtain a profit equal to $E \pi_{i}^{d}\left(\lambda_{M}=1, p^{m} ; s^{-i}\right)=$ $E \pi_{i}\left(\lambda_{B}=1, p^{m} ; s^{-i}\right)+\lambda_{B} \mu_{A} R\left(p^{m}\right)-\phi_{A}$. For this deviation not to be profitable, it must be the case that $\lambda_{B} \mu_{A} R\left(p^{m}\right)-\phi_{A} \leq 0$. Further, in equilibrium a firm entering segment $B$ must be indifferent between charging any price $p \in\left[\bar{p}_{M}, p^{m}\right]$. This implies that $F_{B}(p)=1-\frac{\lambda_{A}}{\lambda_{B}}\left(\frac{R\left(p^{m}\right)-R(p)}{R(p)}\right)$. Furthermore, for any $p \in\left[\underline{p}_{B}, \bar{p}_{M}\right], E \pi_{i}\left(\lambda_{M}=1, p ; s^{-i}\right)=E \pi_{i}\left(\lambda_{B}=1, p ; s^{-i}\right)$, which yields $F_{M}(p)=1-\frac{\phi_{A}}{\lambda_{M} \mu_{A} R(p)}+\frac{\lambda_{B}}{\lambda_{M}}$. The condition $F_{M}\left(\bar{p}_{M}\right)=1$ yields $\lambda_{B} \mu_{A} R\left(\bar{p}_{M}\right)-\phi_{A}=0$, which contradicts the condition above that $\lambda_{B} \mu_{A} R\left(p^{m}\right)-\phi_{A} \leq 0$. Therefore, if an equilibrium exists, then $\bar{p}_{B}=\bar{p}_{M}$, and by the usual arguments $\bar{p}_{B}=\bar{p}_{M}=p^{m}$.

Claim 6: If a positive-profits equilibrium exists, $\underline{p}_{A}<\underline{p}_{M}$.

Proof. Let us assume that $\underline{p}_{A} \geq \underline{p}_{M}$, i.e., $\underline{p}_{M} \leq \underline{p}_{A}<\bar{p}_{A}<\underline{p}_{B}<\bar{p}_{B}=\bar{p}_{M}=p^{m}$. In equilibrium, for any $p \in \sigma_{A}, E \pi_{i}\left(\lambda_{M}=1, p ; s^{-i}\right)=E \pi_{i}\left(\lambda_{A}=1, p ; s^{-i}\right)$. This holds if and only if

$$
R(p) \mu_{B}\left[\lambda_{A}+\lambda_{B}+\lambda_{M}\left(1-F_{M}(p)\right)\right]-\phi_{B}=0
$$

This yields an expression for the prices charged in the entire market $F_{M}(p)=1-\frac{\phi_{B}}{\lambda_{M} \mu_{B} R(p)}+\frac{\lambda_{A}+\lambda_{B}}{\lambda_{M}}$. Further, in equilibrium $E \pi_{i}\left(\lambda_{A}=1, p ; s^{-i}\right)=\lambda_{A} \mu_{B} R\left(p^{m}\right)-\phi_{B}$. Using $F_{M}(p)$, this equality leads to an expression for the prices charged in segment A: $F_{A}(p)=\frac{\phi_{B}-\lambda_{A} \mu_{B}^{2} R\left(p^{m}\right)-\phi_{A} \mu_{B}}{\lambda_{A} \mu_{A} \mu_{B} R(p)}$. Since $F_{A}(p)>$ 0 for all $p \in \sigma_{A}$, it must be the case that $\phi_{B}-\lambda_{A} \mu_{B}^{2} R\left(p^{m}\right)-\phi_{A} \mu_{B}>0$. But then $F_{A}(p)$ would be strictly decreasing in $p$, which cannot happen in equilibrium.

Claim 7: If a positive-profits equilibrium exists, $\underline{p}_{M}=\bar{p}_{A}$.

Proof. The proof of this result is analogous to the proof of Claim 6 and therefore omitted.

This completes the proof of Proposition 1.

Proof of Theorem 2: Let $s^{i}=\left\{\left(\lambda_{j}, F_{j}(p)\right)\right\}_{j \in\{A, B, M\}}$, where $\lambda_{j}, F_{j}(p)$ and $\sigma_{j}, j=A, B, M$ are given in Theorem 2. It is easy to check that the equilibrium conditions $E \pi_{i}\left(\lambda_{j}=1, p ; s^{-i}\right)=$ $\lambda_{A} \mu_{B} R\left(p^{m}\right)-\phi_{B}$ for any $p \in \sigma_{j}$, are satisfied if and only if $\lambda_{j}, F_{j}(p)$ and $\sigma_{j}, j=A, B, M$ take the form presented in the Theorem. 
To prove that the strategies given in Theorem 2 constitute indeed an equilibrium and to prove existence we need to show $(i)$ that firms do not have an incentive to deviate from the strategies prescribed and (ii) that $\lambda_{A}, \lambda_{B}, \lambda_{M} \in(0,1), \lambda_{A}+\lambda_{B}+\lambda_{M}=1$, the lower and upper bounds of the supports of the price distributions satisfy the inequality given in Proposition 1, price distributions are well-behaved and expected profits are strictly positive if $\mu_{A}, \mu_{B}, \phi_{A}$ and $\phi_{B}$ satisfy the condition given in the Theorem.

We start showing that firms cannot profitably deviate. We note that there are various ways in which a firm can deviate. A firm may deviate by advertising a price $p^{d} \notin \sigma_{j}$ in segment $j$. We now prove that this cannot constitute a profitable deviation. Take as given rival's strategy and let firm $i$ deviate by advertising a price $p^{d} \notin \sigma_{A}$ in segment $A$. We have two possibilities. One, let $p^{d} \in\left(\bar{p}_{A}, \underline{p}_{B}\right]$, then using (3), the expected profit to firm $i$ is $E \pi_{i}\left(\lambda_{A}=1, p^{d} ; s^{-i}\right)=\lambda_{B} \mu_{A} R\left(p^{d}\right)+$ $\lambda_{M} \mu_{A} R\left(p^{d}\right)\left(1-F_{M}\left(p^{d}\right)\right)-\phi_{A}$. Using the expression for $F_{M}(p)$ when $p \in\left(\bar{p}_{A}, \underline{p}_{B}\right]$, we note that $E \pi_{i}\left(\lambda_{A}=1, p^{d} ; s^{-i}\right)=\lambda_{A} \mu_{A} \mu_{B}\left(R\left(p^{m}\right)-R\left(p^{d}\right)\right)-\phi_{A}\left(1-\mu_{A}\right)$, which is strictly decreasing in $p^{d}$. Therefore, this deviation is not profitable. Two, let $p^{d} \in \sigma_{B}$; then using (3) and the expression for $F_{M}(p)$ when $p \in \sigma_{B}$ leads to $E \pi_{i}\left(\lambda_{A}=1, p^{d} ; s^{-i}\right)=0$. Thus, firm $i$ cannot increase its profits by advertising a price $p^{d} \notin \sigma_{A}$ in segment $A$. Second, let firm $i$ deviate by advertising a price $p^{d} \notin \sigma_{B}$ in segment $B$; again, we have two possibilities. One, let $p^{d} \in \sigma_{A}$; then, using (4), we observe that $E \pi_{i}\left(\lambda_{B}=1, p^{d} ; s^{-i}\right)=\mu_{B} R\left(p^{d}\right)-\phi_{B}$. Since this expression is strictly increasing in $p^{d}$, firm $i$ will set $p^{d}=\bar{p}_{A}=R^{-1}\left(\phi_{B} / \mu_{B}\right)$; however this yields zero profits. Thus, this deviation is not profitable. Two, let $p^{d} \in\left[\bar{p}_{A}, \underline{p}_{B}\right)$, then using (4) and the expression for $F_{M}(p)$ when $p \in\left[\bar{p}_{A}, \underline{p}_{B}\right)$, the expected profit to firm $i$ is $E \pi_{i}\left(\lambda_{B}=1, p^{d} ; s^{-i}\right)=\lambda_{A} \mu_{B} \mu_{A} R\left(p^{d}\right)+\lambda_{A} \mu_{B} R\left(p^{m}\right)+\phi_{A} \mu_{B}-\phi_{B}$. Since this expression is strictly increasing in $p^{d}$, firm $i$ does not deviate. Third, suppose firm $i$ deviates by advertising a price $p^{d} \notin \sigma_{M}$ in the entire market. Then $p^{d} \in\left[p_{A}, \bar{p}_{A}\right)$. Using (5), the profit to firm $i$ is $E \pi_{i}\left(\lambda_{M}=1, p^{d} ; s^{-i}\right)=\left(1-\lambda_{A}\right) R\left(p^{d}\right)+\lambda_{A} \mu_{B} R\left(p^{d}\right)+\lambda_{A} \mu_{A} R\left(p^{d}\right)\left(1-F_{A}\left(p^{d}\right)\right)-\phi_{A}-\phi_{B}$. Using the expression for $F_{A}(p)$ obtained above this profit can be rewritten as $E \pi_{i}\left(\lambda_{M}=1, p^{d} ; s^{-i}\right)=$ $\mu_{B} R\left(p^{d}\right)+\left(1-\lambda_{A}\right) \mu_{A} R\left(\bar{p}_{A}\right)-\phi_{A}-\phi_{B}$, which is strictly increasing in $p^{d}$. Hence, this deviation is not profitable. We now observe that a firm may also deviate by advertising a price $p^{d} \in \sigma_{j}$ in segment $j^{\prime} \neq j$. This type of deviation is however ruled out by the cases above where a firm charges a price $p^{d} \notin \sigma_{j^{\prime}}$ in $j^{\prime}$. Finally, a firm may also deviate by advertising a price $p^{d} \notin \sigma_{j}$ in $j^{\prime} \neq j$, but 
these deviations are also ruled out by the previous arguments. This completes the proof of $(i)$.

We now show $(i i)$. We start noting that $\lambda_{A}>0$ since $\phi_{B} / \mu_{B} \geq \phi_{A} / \mu_{A}>\phi_{A}$. Moreover, $\lambda_{A}<1$ if and only if $\phi_{A} / \mu_{A}>\left(\phi_{B} / \mu_{B}-R\left(p^{m}\right)\right) / \mu_{A}$, which is always satisfied because $\phi_{B} / \mu_{B}-R\left(p^{m}\right)<0$. It is readily seen that $0<\lambda_{B}<1$. We note that $\lambda_{M}=1-\lambda_{A}-\lambda_{B}$ and that is strictly positive if and only if

$$
\frac{\phi_{A}}{\mu_{A}}\left(\mu_{B} R\left(p^{m}\right)\left(\mu_{B}-\mu_{A}\right)+\phi_{B} \mu_{A}\right)<\mu_{B} R\left(p^{m}\right)\left(\mu_{B} R\left(p^{m}\right)-\phi_{B}\right) .
$$

Note that if the LHS of (9) is negative, the condition holds; otherwise, $\lambda_{M}>0$ requires

$$
\frac{\phi_{A}}{\mu_{A}}<\frac{\mu_{B} R\left(p^{m}\right)\left(\mu_{B} R\left(p^{m}\right)-\phi_{B}\right)}{\mu_{B} R\left(p^{m}\right)\left(\mu_{B}-\mu_{A}\right)+\phi_{B} \mu_{A}}
$$

We now observe that expected profit $E \pi=\lambda_{A} \mu_{B} R\left(p^{m}\right)-\phi_{B}$ is strictly positive if and only if

$$
\frac{\phi_{A}}{\mu_{A}}<\frac{\phi_{B}}{\mu_{B}}\left(1-\frac{\phi_{B}}{\mu_{B} R\left(p^{m}\right)}\right)
$$

which is the condition in the Theorem. We now note that $\underline{p}_{A}<\underline{p}_{M}$ because $\lambda_{A}>0$. Further, $\underline{p}_{M}<\underline{p}_{B}$ if and only if (11) holds. Furthermore, $\underline{p}_{B}>0$ if and only if $\lambda_{A}>\lambda_{B}$, or

$$
\frac{\phi_{A}}{\mu_{A}}<\frac{\phi_{B}}{\mu_{B}}\left(\frac{R\left(p^{m}\right) \mu_{B}}{\mu_{B} R\left(p^{m}\right)+\phi_{B} \mu_{A}}\right)
$$

We now show that if condition (11) holds, then (10) and (12) also hold. We prove this by showing that the RHS of (11) is lower than the RHS of (10) and (12). Consider first (10). We need to show that

$$
\begin{aligned}
\frac{\phi_{B}}{\mu_{B}}\left(1-\frac{\phi_{B}}{\mu_{B} R\left(p^{m}\right)}\right) & <\frac{\mu_{B} R\left(p^{m}\right)\left(\mu_{B} R\left(p^{m}\right)-\phi_{B}\right)}{\mu_{B} R\left(p^{m}\right)\left(\mu_{B}-\mu_{A}\right)+\phi_{B} \mu_{A}} \\
\frac{1}{\mu_{B}}\left(\frac{\phi_{B}}{\mu_{B} R\left(p^{m}\right)}\right) & <\frac{\mu_{B} R\left(p^{m}\right)}{\mu_{B} R\left(p^{m}\right)\left(\mu_{B}-\mu_{A}\right)+\phi_{B} \mu_{A}}
\end{aligned}
$$


Since $\phi_{B}<\mu_{B} R\left(p^{m}\right)$, it is sufficient to show that

$$
\begin{aligned}
\frac{1}{\mu_{B}} & <\frac{\mu_{B} R\left(p^{m}\right)}{\mu_{B} R\left(p^{m}\right)\left(\mu_{B}-\mu_{A}\right)+\phi_{B} \mu_{A}} \\
\mu_{B} R\left(p^{m}\right)\left(\mu_{B}-\mu_{A}\right)+\phi_{B} \mu_{A} & <\mu_{B}^{2} R\left(p^{m}\right) \\
-\mu_{A}\left(\mu_{B} R\left(p^{m}\right)-\phi_{B}\right) & <0 .
\end{aligned}
$$

Similarly, consider (12). We need to show that

$$
\begin{aligned}
\frac{\mu_{B} R\left(p^{m}\right)-\phi_{B}}{\mu_{B} R\left(p^{m}\right)} & <\frac{R\left(p^{m}\right) \mu_{B}}{\mu_{B} R\left(p^{m}\right)+\phi_{B} \mu_{A}} \\
-\mu_{B} R\left(p^{m}\right) \phi_{B}\left(1-\mu_{A}\right)-\phi_{B}^{2} \mu_{A} & <0 .
\end{aligned}
$$

It remains to show that the price distributions are increasing in $p$. This is readily seen for $F_{A}(p)$ and $F_{M}(p)$; for $F_{B}(p)$, this follows from the fact that $\lambda_{A}>\lambda_{B}$. This completes the proof that this equilibrium exists whenever $\frac{\phi_{A}}{\mu_{A}} \mu_{B} R\left(p^{m}\right)<\frac{\phi_{B}}{\mu_{B}}\left(\mu_{B} R\left(p^{m}\right)-\phi_{B}\right)$. Proposition 1 implies that this is the unique positive-profits equilibrium. This completes the proof of Theorem 2.

For later reference, let assume, without loss of generality, that $\phi_{A}=\gamma R\left(p^{m}\right)$, with $\gamma \in\left(0, \mu_{A}\right)$ and $\phi_{B}=\beta \phi_{A}$, with $\beta \in\left[\mu_{B} / \mu_{A}, \mu_{B} / \gamma\right)$. We note that the existence condition for the positive-profits equilibrium can be rewritten as $\gamma<\mu_{B}\left(\mu_{A} \beta-\mu_{B}\right) / \beta^{2} \mu_{A}$.

Proof of Proposition 2: Given that the $\sigma_{A}, \sigma_{B}$ and $\sigma_{M}$ must satisfy Proposition 1, we only need to show that $F_{M}(p)>F_{B}(p)$ for all $p \in \sigma_{B}$. Using the expressions above, it suffices to show that $\lambda_{A}\left(1-\lambda_{A}\right)>\lambda_{B}$. Using the formulas for $\lambda_{A}$ and $\lambda_{B}$ given in Theorem 2 , one gets that $\lambda_{A}\left(1-\lambda_{A}\right)>\lambda_{B}$ if and only if

$$
\begin{aligned}
\frac{\gamma\left(\beta-\mu_{B}\right)}{\mu_{B}^{2}+\gamma \beta \mu_{A}}\left(1-\frac{\gamma\left(\beta-\mu_{B}\right)}{\mu_{B}^{2}+\gamma \beta \mu_{A}}\right) & >\frac{\gamma}{\mu_{A}} \\
\text { or } \gamma\left(\gamma \mu_{A}+\mu_{B}\right) \frac{\mu_{B}^{2}(1+\beta)+\beta^{2} \gamma \mu_{A}-\beta \mu_{B}}{\left(\mu_{B}^{2}+\beta \gamma-\beta \gamma \mu_{B}\right)^{2} \mu_{A}} & >0 .
\end{aligned}
$$

Isolating $\gamma$ we obtain

$$
\gamma<\frac{\beta \mu_{B}-\mu_{B}^{2}(1+\beta)}{\beta^{2} \mu_{A}}=\frac{\mu_{B}\left(\beta-\mu_{B}(1+\beta)\right)}{\beta^{2} \mu_{A}}=\frac{\mu_{B}\left(\beta \mu_{A}+\mu_{B}\right)}{\beta^{2} \mu_{A}}
$$


which is always satisfied when the condition in Theorem 2 holds.

Proof of Proposition 3. (1) First, $\frac{\partial \lambda_{A}}{\partial \gamma}=\frac{\mu_{B}^{2}\left(\beta-\mu_{B}\right)}{\left(\mu_{B}^{2}+\mu_{A} \beta \gamma\right)^{2}}$ which is strictly positive given the equilibrium condition. Second, $\frac{\partial \lambda_{B}}{\partial \gamma}=\frac{\gamma}{\mu_{A}}>0$; as a consequence $\frac{\partial \lambda_{M}}{\partial \gamma}<0$. Third, we claim that an increase in $\gamma$ widens $\sigma_{B}$. To see this note that $R\left(\underline{p}_{B}\right)=R\left(p^{m}\right)\left(1-\frac{\lambda_{B}}{\lambda_{A}}\right)$ and $\frac{\lambda_{B}}{\lambda_{A}}=\frac{\mu_{B}^{2}+\mu_{A} \beta \gamma}{\mu_{A}(\beta-1)}$. Since an increase in $\gamma$ raises $\frac{\lambda_{B}}{\lambda_{A}}$, it follows that $\frac{\partial R\left(\underline{p}_{B}\right)}{\partial \gamma}<0$; thus the claim follows. Fourth, an increase in $\gamma$ widens $\sigma_{A}$ if and only if $\frac{\partial\left(\frac{R\left(\bar{p}_{A}\right)}{R\left(\underline{p}_{A}\right)}\right)}{\partial \gamma}=\frac{\partial\left(\frac{1}{1-\lambda_{A}}\right)}{\partial \gamma}>0$; since $\frac{\partial \lambda_{A}}{\partial \gamma}>0$ this is always satisfied. Fifth, an increase in $\gamma$ narrows $\sigma_{M}$ because $\frac{\partial R\left(\underline{p}_{M}\right)}{\partial \gamma}=\frac{\beta R\left(p^{m}\right)}{\mu_{B}}>0$. Sixth, equilibrium profits are $E \pi=R\left(p^{m}\right)\left(\lambda_{A} \mu_{B}-\beta \gamma\right)$ and in equilibrium $\gamma \in\left(0, \frac{\mu_{B}\left(\beta \mu_{A}-\mu_{B}\right)}{\beta^{2} \mu_{A}}\right)$. Further, $\frac{\partial E \pi}{\partial \gamma}=R\left(p^{m}\right)\left(\frac{\partial \lambda_{A}}{\partial \gamma}-\beta\right)$ and using the expression for $\frac{\partial \lambda_{A}}{\partial \gamma}$ derived above, we note that $\frac{\partial E \pi}{\partial \gamma}>0$ if and only if $\mu_{B}^{3}\left(\beta-\mu_{B}\right)-\beta\left(\mu_{B}^{2}+\mu_{A} \beta \gamma\right)^{2}>0$; otherwise $\frac{\partial E \pi}{\partial \gamma}<0$. It is readily seen that the expression $\mu_{B}^{3}\left(\beta-\mu_{B}\right)-\beta\left(\mu_{B}^{2}+\mu_{A} \beta \gamma\right)^{2}$ is decreasing in $\gamma$ and strictly positive for $\gamma=0$. Moreover, the expression $\mu_{B}^{3}\left(\beta-\mu_{B}\right)-\beta\left(\mu_{B}^{2}+\mu_{A} \beta \gamma\right)^{2}$ is negative for $\gamma=\frac{\mu_{B}\left(\beta \mu_{A}-\mu_{B}\right)}{\beta^{2} \mu_{A}}$ whenever $\left(\beta-\mu_{B}\right)\left(\mu_{B}-\beta \mu_{A}\right)<0$, which is always satisfied in equilibrium. Finally, we note that as $\gamma \rightarrow 0, \lambda_{A}$ and $\lambda_{B}$ converge to zero which implies that $\lambda_{M} \rightarrow 1$. In addition $\underline{p}_{M}$ goes to zero and $F_{M}(p) \rightarrow 1$. This completes the proof of point (1). We now prove (2). First, $\frac{\partial \lambda_{A}}{\partial \beta}=\frac{\gamma \mu_{B}\left(\mu_{B}+\mu_{A} \gamma\right)}{\left(\mu_{B}^{2}+\mu_{A} \beta \gamma\right)^{2}}$, which is strictly positive. Second, $\frac{\partial \lambda_{B}}{\partial \beta}=0$. These two points imply that $\frac{\partial \lambda_{M}}{\partial \beta}<0$. Third, since $R\left(\underline{p}_{B}\right)=R\left(p^{m}\right)\left(1-\frac{\lambda_{B}}{\lambda_{A}}\right), \frac{\partial \lambda_{A}}{\partial \beta}>0$ and $\frac{\partial \lambda_{B}}{\partial \beta}=0$, it follows that an increase in $\beta$ narrows $\sigma_{B}$, i.e., $\frac{\partial R\left(\underline{p}_{B}\right)}{\partial \beta}>0$. Fourth, like in (1), since $\frac{\partial \lambda_{A}}{\partial \beta}>0$, it follows that an increase in $\beta$ widens $\sigma_{A}$. Fifth, an increase in $\beta$ narrows $\sigma_{M}$ because $\frac{\partial R\left(\underline{p}_{M}\right)}{\partial \beta}=\frac{\gamma R\left(p^{m}\right)}{\mu_{B}}>0$. Finally, $\frac{\partial E \pi}{\partial \beta}=R\left(p^{m}\right)\left(\frac{\partial \lambda_{A}}{\partial \beta} \mu_{B}-\gamma\right)$; using the expression for $\frac{\partial \lambda_{A}}{\partial \beta}$ derived above we obtain that $\frac{\partial E \pi}{\partial \beta}>0$ if and only if $\mu_{B}^{2}\left(\mu_{B}+\mu_{A} \gamma\right)-\left(\mu_{B}^{2}+\beta \gamma \mu_{A}\right)^{2}>0$, otherwise it is negative. Let $\psi(\gamma)=\mu_{B}^{2}\left(\mu_{B}+\mu_{A} \gamma\right)-\left(\mu_{B}^{2}+\beta \gamma \mu_{A}\right)^{2}$. We observe that $\frac{\partial \psi}{\partial \gamma}=\mu_{B}^{2} \mu_{A}(1-2 \beta)-2 \beta^{2} \gamma \mu_{A}^{2}<0$; to see this note that in equilibrium $\lambda_{A}<1$ if and only if $\gamma<\mu_{B} /(\beta-1)$, and since $\gamma$ must be strictly positive in equilibrium it follows that $\beta>1$. Further, we observe that $\psi(\gamma)>0$ for $\beta<2 \mu_{B} / \mu_{A}$; if instead $\beta>2 \mu_{B} / \mu_{A}$ then $\psi(\gamma)>0$ for $\gamma=0$ and negative for $\gamma=\mu_{B}\left(\beta \mu_{A}-\mu_{B}\right) / \beta^{2} \mu_{A}$. This competes the proof of point (2) of the Proposition. We conclude with (3). First, we claim that $\frac{\partial \lambda_{A}}{\partial \mu_{B}}<0$. We note that $\frac{\partial \lambda_{A}}{\partial \mu_{B}}=\frac{\gamma\left[\beta \gamma(\beta-1)-\mu_{B}\left(2 \beta-\mu_{B}\right)\right]}{\left(\mu_{B}^{2}+\mu_{A} \beta \gamma\right)^{2}}<0$ if and only if $\beta \gamma(\beta-1)-\mu_{B}\left(2 \beta-\mu_{B}\right)<0$; this condition is satisfied if and only if $\gamma<\frac{\mu_{B}\left(2 \beta-\mu_{B}\right)}{\beta(\beta-1)}$, which holds given the existence condition in Theorem 2 ; hence, the claim follows. Second, $\frac{\partial \lambda_{B}}{\partial \mu_{B}}=\frac{\gamma}{\mu_{A}}>0$. Third, since $R\left(\underline{p}_{B}\right)=R\left(p^{m}\right)\left(1-\frac{\lambda_{B}}{\lambda_{A}}\right)$, $\frac{\partial \lambda_{A}}{\partial \mu_{B}}<0$ and $\frac{\partial \lambda_{B}}{\partial \mu_{B}}>0$, it follows that an increase in $\mu_{B}$ widens $\sigma_{B}$, i.e., $\frac{\partial R\left(\underline{p}_{B}\right)}{\partial \mu_{B}}<0$. Fourth, since 
$\frac{\partial \lambda_{A}}{\partial \mu_{B}}<0$, an increase in $\mu_{B}$ narrows $\sigma_{A}$. Fifth, since $R\left(\underline{p}_{M}\right)=\frac{\beta \gamma R\left(p^{m}\right)}{\mu_{B}}$ an increase in $\mu_{B}$ widens $\sigma_{M}$. Finally, $\frac{\partial E \pi}{\partial \mu_{B}}=R\left(p^{m}\right)\left(\frac{\partial \lambda_{A}}{\partial \mu_{B}} \mu_{B}-\lambda_{A}\right)$; using the expression for $\frac{\partial \lambda_{A}}{\partial \mu_{B}}$ derived above and the expression for $\lambda_{A}$ we obtain that $\frac{\partial E \pi}{\partial \beta}>0$ if and only if $\gamma\left(\beta-\mu_{B}\right)-\mu_{B}\left(\mu_{B}+\gamma \mu_{A}\right)>0$; otherwise it is negative. Let $\psi(\gamma)=\gamma\left(\beta-\mu_{B}\right)-\mu_{B}\left(\mu_{B}+\gamma \mu_{A}\right)$. We observe that $\frac{\partial \psi}{\partial \gamma}=\beta-\mu_{B}-\mu_{B} \mu_{A}>0$ if and only if $\beta>\mu_{B}\left(1+\mu_{A}\right)$; since in equilibrium $\beta>1$, this condition is always satisfied. Further, $\psi(\gamma=0)=-\mu_{B}^{2}<0$. Furthermore we observe that when $\psi\left(\gamma=\mu_{B}\left(\beta \mu_{A}-\mu_{B}\right) / \beta^{2} \mu_{A}\right)>0$ if and only if $\beta>\mu_{B}\left(1+\mu_{A}\right) / \mu_{A}^{2}$; otherwise it is negative. This competes the proof of point (3) of the Proposition. The proof is now complete.

Proof of Theorem 3: Let $\lambda_{j} \in(0,1), j \in\{A, B, M\}$ and $\lambda_{O} \in[0,1)$ and let $\underline{p}_{A}<\bar{p}_{A}=$ $\underline{p}_{B}=\underline{p}_{M}<\bar{p}_{B}=\bar{p}_{M}=p^{m}$. We first note that since $p^{m} \in \sigma_{B}=\sigma_{M}$ in equilibrium a firm must be indifferent between advertising $p^{m}$ in segment $B$ or advertising the same price in the entire market, i.e., $E \pi_{i}\left(\lambda_{B}=1, p^{m} ; s^{-i}\right)=E \pi_{i}\left(\lambda_{M}=1, p^{m} ; s^{-i}\right)=0$. Solving these two conditions we obtain $\lambda_{O}+\lambda_{A}=\phi_{B} / \mu_{B} R\left(p^{m}\right)$ and $\lambda_{O}+\lambda_{B}=\phi_{A} / \mu_{A} R\left(p^{m}\right)$. Similarly, since $\bar{p}_{A} \in \sigma_{j}$, $j=A, B, M$, in equilibrium it must be the case that $E \pi_{i}\left(\lambda_{j}=1, \bar{p}_{A} ; s^{-i}\right)=0$, for any $j=A, B, M$. Solving these conditions we obtain the frequency with which a firm advertises in segment $A, \lambda_{A}=$ $\left(\phi_{B} \mu_{A}-\phi_{A} \mu_{B}\right) / \phi_{B} \mu_{A}$, and the upper bound $\bar{p}_{A}=R^{-1}\left(\phi_{B} / \mu_{B}\right)$. Plugging $\lambda_{A}$ into the expressions above yields

$$
\lambda_{O}=\frac{\phi_{B}^{2} \mu_{A}-\mu_{B} R\left(p^{m}\right)\left(\phi_{B} \mu_{A}-\phi_{A} \mu_{B}\right)}{\phi_{B} \mu_{B} \mu_{A} R\left(p^{m}\right)} \text { and } \lambda_{B}=\frac{\left(\phi_{B} \mu_{A}-\phi_{A} \mu_{B}\right)\left(\mu_{B} R\left(p^{m}\right)-\phi_{B}\right)}{\phi_{B} \mu_{A} \mu_{B} R\left(p^{m}\right)}
$$

and $\lambda_{M}$ simply follows from $\lambda_{M}=1-\lambda_{O}-\lambda_{A}-\lambda_{B}$. Second, the expected profit to a firm advertising a price $p \in \sigma_{B}=\sigma_{M}$ in segment $B$ is $E \pi_{i}\left(\lambda_{B}=1, p ; s^{-i}\right)=R(p) \mu_{B}\left[\lambda_{O}+\lambda_{A}+\lambda_{B}\left(1-F_{B}(p)\right)+\right.$ $\left.\lambda_{M}\left(1-F_{M}(p)\right)\right]-\phi_{B}$; similarly the expected profit to a firm advertising the same price in the entire market is $E \pi_{i}\left(\lambda_{M}=1, p ; s^{-i}\right)=E \pi_{i}\left(\lambda_{B}=1, p ; s^{-i}\right)+R(p) \mu_{A}\left[\lambda_{O}+\lambda_{B}+\lambda_{M}\left(1-F_{M}(p)\right)\right]-\phi_{A}$. In equilibrium it must be the case that $E \pi_{i}\left(\lambda_{j}=1, p ; s^{-i}\right)=0$, for $j=B, M$. Solving these conditions yields $F_{B}(p)=F_{M}(p)=1-\frac{\phi_{B}}{R\left(p^{m}\right) \mu_{B}-\phi_{B}} \frac{R\left(p^{m}\right)-R(p)}{R(p)}$. Third, for any price $p \in \sigma_{A}$ it must be the case that $E \pi_{i}\left(\lambda_{A}=1, p ; s^{-i}\right)=R(p) \mu_{A}\left[1-\lambda_{A}+\lambda_{A}\left(1-F_{A}(p)\right)\right]-\phi_{A}=0$, which yields the equilibrium price distribution $F_{A}(p)=1-\frac{1}{\lambda_{A}}\left(\frac{\phi_{A}}{R(p) \mu_{A}}-\left(1-\lambda_{A}\right)\right)$. Using this expression we can determine $\underline{p}_{A}$ by solving $F_{A}\left(\underline{p}_{A}\right)=0$, which yields $\underline{p}_{A}=R^{-1}\left(\phi_{A} / \mu_{A}\right)$. 
To prove that this characterization constitutes indeed an equilibrium and to prove existence we need to show $(i)$ that firms do not have an incentive to deviate from the strategies prescribed in the Theorem; (ii) that the lower and upper bounds of the supports of the price distributions satisfy $\underline{p}_{A}<\bar{p}_{A}=\underline{p}_{B}=\underline{p}_{M}<\bar{p}_{B}=\bar{p}_{M}=p^{m}$ and that price distributions are well-behaved; and (iii) that $\lambda_{j} \in(0,1), j \in\{A, B, M\}$ and $\lambda_{O} \in[0,1)$.

It is easy to check that $(i)$ and $(i i)$ are satisfied. We now prove $(i i i)$. First, it is readily seen that $\lambda_{A}, \lambda_{B}, \lambda_{M} \in(0,1)$ and $\lambda_{O}<1$; moreover, inspection of the expression for $\lambda_{O}$ reveals that $\lambda_{O} \geq 0$ if and only if $\phi_{A} / \mu_{A} \geq\left(\phi_{B} / \mu_{B}\right)\left(1-\phi_{B} / R\left(p^{m}\right) \mu_{B}\right)$. This completes the proof of the Theorem 2 .

Proof of Proposition 4: Firms' payoffs from the different advertising and pricing strategies are given by:

$$
\begin{aligned}
E \pi_{i}\left(\lambda_{A}=\right. & \left.1, p \in \sigma_{A} ; s^{-i}\right)=R(p)\left(\mu_{A}+\mu_{A B}\right)\left[\lambda_{A}\left(1-F_{A}(p)\right)+\lambda_{B}+\lambda_{M}\right]-\phi_{A} \\
E \pi_{i}\left(\lambda_{B}=\right. & \left.1, p \in \sigma_{B} ; s^{-i}\right)=R(p)\left[\mu_{B} \lambda_{A}+\lambda_{B}\left(\mu_{B}+\mu_{A B}\right)\left(1-F_{B}(p)\right)\right. \\
& \left.+\lambda_{M}\left(\mu_{B}+\mu_{A B}\right)\left(1-F_{M}(p)\right)\right]-\phi_{B} \\
E \pi_{i}\left(\lambda_{M}=\right. & \left.1, p \in \sigma_{M} \cap \sigma_{B} ; s^{-i}\right)=R(p)\left[\mu_{B} \lambda_{A}+\lambda_{B}\left[\mu_{A}+\left(\mu_{B}+\mu_{A B}\right)\left(1-F_{B}(p)\right)\right]\right. \\
& \left.+\lambda_{M}\left(1-F_{M}(p)\right)\right]-\phi_{A}-\phi_{B}
\end{aligned}
$$

Consider the strategy profile specified in Proposition 4. We note that this is the unique strategy profile that satisfies the conditions given in Proposition 1. To prove that these strategies constitute a positive-profits equilibrium and to prove existence we proceed as in the proof of Theorem 2.

We start showing that firms cannot profitably deviate. Assume, without loss of generality, that firm 2 follows the strategy prescribed in the Proposition. Consider that firm 1 deviates by advertising a price $p^{d} \notin \sigma_{A}$ in segment $A$. Assume first $p^{d} \in\left(\bar{p}_{A}, \underline{p}_{B}\right]$, then $E \pi_{1}^{d}\left(\lambda_{A}=1, p^{d} ; s^{2}\right)=$ $\lambda_{B}\left(1-\mu_{B}\right) R\left(p^{d}\right)+\lambda_{M}\left(1-\mu_{B}\right) R\left(p^{d}\right)\left(1-F_{M}\left(p^{d}\right)\right)-\phi_{A}$. Using the expression for $F_{M}(p)$ in the Proposition yields $E \pi_{1}^{d}\left(\lambda_{A}=1, p^{d} ; s^{2}\right)=\left(1-\mu_{B}\right) \lambda_{A} \mu_{B}\left(R\left(p^{m}\right)-R\left(p^{d}\right)\right)-\phi_{A} \mu_{B}$, which is strictly decreasing in $p^{d}$. Therefore this deviation is not profitable. Suppose now that $p^{d} \in$ $\sigma_{B}$; then deviating profits are $E \pi_{1}^{d}\left(\lambda_{A}=1, p^{d} ; s^{2}\right)=\lambda_{B} \mu_{A} R\left(p^{d}\right)+\lambda_{B} \mu_{A B} R\left(p^{d}\right)\left(1-F_{B}\left(p^{d}\right)\right)+$ 
$\lambda_{M}\left(1-\mu_{B}\right) R\left(p^{d}\right)\left(1-F_{M}\left(p^{d}\right)\right)-\phi_{A}$. Using the expressions for $F_{B}(p)$ and $F_{M}(p)$ in the Proposition we obtain $E \pi_{1}^{d}\left(\lambda_{A}=1, p^{d} ; s^{2}\right)=\frac{\mu_{A B}}{1-\mu_{A}} \lambda_{A} \mu_{B}\left(R\left(p^{m}\right)-R\left(p^{d}\right)\right)$. This profit is decreasing in $p^{d}$ and therefore the most profitable deviation consists of setting $p^{d}=\underline{p}_{B}$. However, we have shown above that a firm advertising $\underline{p}_{B}$ in segment $A$ gets lower profits than in equilibrium. In summary, a firm that advertises in segment $A$ does not gain by charging a price $p^{d} \notin \sigma_{A}$.

Second, consider that firm 1 deviates by advertising $p^{d} \notin \sigma_{B}$ in segment $B$ Consider first that $p^{d} \in\left[\bar{p}_{A}, \underline{p}_{B}\right)$; then deviating profits are $E \pi_{1}^{d}\left(\lambda_{B}=1, p^{d} ; s^{2}\right)=\lambda_{A} \mu_{B} R\left(p^{d}\right)+\lambda_{B}\left(1-\mu_{A}\right) R\left(p^{d}\right)+$ $\lambda_{M}\left(1-\mu_{A}\right) R\left(p^{d}\right)\left(1-F_{M}\left(p^{d}\right)\right)-\phi_{B}$. Using the expression for $F_{M}(p)$ in the Proposition we obtain $E \pi_{1}^{d}\left(\lambda_{B}=1, p^{d} ; s^{2}\right)=\lambda_{A} \mu_{B}\left(1-\mu_{A}\right) R\left(p^{m}\right)+\lambda_{A} \mu_{B} \mu_{A} R\left(p^{d}\right)+\phi_{A}\left(1-\mu_{A}\right)-\phi_{B}$, which is strictly increasing in $p^{d}$ and therefore this deviation is not profitable. Assume now that $p^{d} \in \sigma_{A}$, then $E \pi_{1}^{d}\left(\lambda_{B}=1, p^{d} ; s^{2}\right)=\lambda_{A} \mu_{B} R\left(p^{d}\right)+\lambda_{A} \mu_{A B} R\left(p^{d}\right)\left(1-F_{A}\left(p^{d}\right)\right)+\left(1-\lambda_{A}\right)\left(1-\mu_{A}\right) R\left(p^{d}\right)-\phi_{B}$. Using the expression for $F_{A}(p)$ in the Proposition we obtain $E \pi_{1}^{d}\left(\lambda_{B}=1, p^{d} ; s^{2}\right)=\mu_{A B}\left(1-\lambda_{A}\right) R\left(\bar{p}_{A}\right)+$ $\mu_{B} R\left(p^{d}\right)-\phi_{B}$. Since this expression is strictly increasing in $p^{d}$, the most profitable deviation consists of setting $p^{d}=\bar{p}_{A}$, which is not profitable. Thus, firm 1 advertising in segment $B$ does not gain by charging a price $p^{d} \notin \sigma_{B}$.

Finally, consider that firm 1 deviates by advertising a price $p^{d} \notin \sigma_{M}$ in the entire market, i.e., $p^{d} \in\left[\underline{p}_{A}, \bar{p}_{A}\right)$. The deviating profit is $E \pi_{1}^{d}\left(\lambda_{M}=1, p^{d} ; s^{2}\right)=\lambda_{A} \mu_{B} R\left(p^{d}\right)+\lambda_{A}\left(1-\mu_{B}\right) R\left(p^{d}\right)(1-$ $\left.F_{A}\left(p^{d}\right)\right)+\left(1-\lambda_{A}\right) R\left(p^{d}\right)-\phi_{A}-\phi_{B}$. Using the expression for $F_{A}(p)$ above, we obtain $E \pi_{1}^{d}\left(\lambda_{M}=1, p^{d} ; s^{2}\right)=$ $\left(1-\mu_{B}\right)\left(1-\lambda_{A}\right) R\left(\bar{p}_{A}\right)+R\left(p^{d}\right) \mu_{B}-\phi_{A}-\phi_{B}$, which is strictly increasing in $p^{d}$. Thus firm 1 advertising in the entire market does not gain by charging a price $p^{d} \notin \sigma_{M}$.

We now show $\lambda_{j} \in(0,1), \lambda_{A}+\lambda_{B}+\lambda_{M}=1, j \in\{A, B, M\}$. The same arguments as those in the proof of Theorem 2 show that $\lambda_{A} \in(0,1)$ and $\lambda_{B} \in(0,1)$. It is easy to see that $\lambda_{M}=1-\lambda_{A}-\lambda_{B}$. We note that $\lambda_{M}>0$ if and only if

$$
\frac{\phi_{A}}{\mu_{A}}\left(\mu_{B} R\left(p^{m}\right)\left(\mu_{B}-\mu_{A}\right)+\phi_{B}\left(1-\mu_{B}\right)\right)<\mu_{B} R\left(p^{m}\right)\left(\mu_{B} R\left(p^{m}\right)-\phi_{B}\right)
$$

If the LHS of (16) is negative, the condition holds trivially; otherwise, it must be the case that

$$
\frac{\phi_{A}}{\mu_{A}}<\frac{\mu_{B} R\left(p^{m}\right)\left(\mu_{B} R\left(p^{m}\right)-\phi_{B}\right)}{\mu_{B} R\left(p^{m}\right)\left(\mu_{B}-\mu_{A}\right)+\phi_{B}\left(1-\mu_{B}\right)} .
$$


We now observe that expected profit $E \pi=\lambda_{A} \mu_{B} R\left(p^{m}\right)-\phi_{B}$ is strictly positive if and only if

$$
\frac{\phi_{A}}{\mu_{A}}<\frac{\left(1-\mu_{B}\right)}{\mu_{A}} \frac{\phi_{B}}{\mu_{B}}\left(1-\frac{\phi_{B}}{\mu_{B} R\left(p^{m}\right)}\right)
$$

Note that $\underline{p}_{A}>0$ trivially holds and $\underline{p}_{M}>0$ if $\lambda_{A} \mu_{B} R\left(p^{m}\right)-\phi_{B}>0$, which holds whenever (18) is satisfied. Further, $\underline{p}_{A}<\underline{p}_{M}$ because $\lambda_{A}>0$. Furthermore, $\underline{p}_{M}<\underline{p}_{B}$ if and only if $\left(1-\lambda_{A}\right)\left(\lambda_{A} \mu_{B}-\right.$ $\left.\lambda_{B}\left(1-\mu_{A}\right)\right)>\lambda_{A} \lambda_{B} \mu_{B}$. Using the expressions for $\lambda_{A}$ and $\lambda_{B}$ above, this condition boils down to

$$
\frac{\phi_{A}}{\mu_{A}}<\frac{\phi_{B}\left(\mu_{B} R\left(p^{m}\right)-\phi_{B}\right)}{\mu_{B}\left(\mu_{B} R\left(p^{m}\right)-\phi_{B}\right)+\phi_{B}\left(1-\mu_{A}\right)} .
$$

Finally, we note that if (19) holds, then $\underline{p}_{B}>0$. We now show that if condition (19) holds, then (17) and (18) also hold. We prove this by showing that the RHS of (19) is lower than the RHS of the other conditions. Consider first (17). We need to show that

$$
\begin{gathered}
\frac{\phi_{B}\left(\mu_{B} R\left(p^{m}\right)-\phi_{B}\right)}{\mu_{B}\left(\mu_{B} R\left(p^{m}\right)-\phi_{B}\right)+\phi_{B}\left(1-\mu_{A}\right)}>\frac{\mu_{B} R\left(p^{m}\right)\left(\mu_{B} R\left(p^{m}\right)-\phi_{B}\right)}{\mu_{B} R\left(p^{m}\right)\left(\mu_{B}-\mu_{A}\right)+\phi_{B}\left(1-\mu_{B}\right)} \\
\frac{\phi_{B}}{\mu_{B}\left(\mu_{B} R\left(p^{m}\right)-\phi_{B}\right)+\phi_{B}\left(1-\mu_{A}\right)}<\frac{\mu_{B} R\left(p^{m}\right)}{\mu_{B} R\left(p^{m}\right)\left(\mu_{B}-\mu_{A}\right)+\phi_{B}\left(1-\mu_{B}\right)}
\end{gathered}
$$

Since $\phi_{B}<\mu_{B} R\left(p^{m}\right)$, it is sufficient to show that the denominator of the LHS is larger than the denominator of the RHS:

$$
\begin{aligned}
\mu_{B}\left(\mu_{B} R\left(p^{m}\right)-\phi_{B}\right)+\phi_{B}\left(1-\mu_{A}\right) & >\mu_{B} R\left(p^{m}\right)\left(\mu_{B}-\mu_{A}\right)+\phi_{B}\left(1-\mu_{B}\right) \\
\phi_{B}\left(1-\mu_{A}-\mu_{B}-1+\mu_{B}\right) & >\mu_{B} R\left(p^{m}\right)\left(\mu_{B}-\mu_{A}-\mu_{B}\right) \\
-\mu_{A} \phi_{B} & >-\mu_{A} \mu_{B} R\left(p^{m}\right) \\
\phi_{B} & <\mu_{B} R\left(p^{m}\right)
\end{aligned}
$$

which holds always. Second, consider (18); it must be the case that

$$
\begin{aligned}
\frac{\phi_{B}\left(\mu_{B} R\left(p^{m}\right)-\phi_{B}\right)}{\mu_{B}\left(\mu_{B} R\left(p^{m}\right)-\phi_{B}\right)+\phi_{B}\left(1-\mu_{A}\right)} & <\frac{\left(1-\mu_{B}\right)}{\mu_{A}} \frac{\phi_{B}}{\mu_{B}}\left(1-\frac{\phi_{B}}{\mu_{B} R\left(p^{m}\right)}\right) \\
\frac{1}{\mu_{B}\left(\mu_{B} R\left(p^{m}\right)-\phi_{B}\right)+\phi_{B}\left(1-\mu_{A}\right)} & <\frac{\left(1-\mu_{B}\right)}{\mu_{A}} \frac{1}{\mu_{B}} \frac{1}{\mu_{B} R\left(p^{m}\right)}
\end{aligned}
$$


Using the fact that $1-\mu_{B}=\mu_{A}+\mu_{A B}$, this inequality can be rewritten as follows:

$$
\begin{aligned}
\mu_{A} \mu_{B}^{2} R\left(p^{m}\right) & <\left(\mu_{A}+\mu_{A B}\right) \mu_{B}\left(\mu_{B} R\left(p^{m}\right)-\phi_{B}\right)+\phi_{B}\left(1-\mu_{B}\right)\left(1-\mu_{A}\right) \\
0 & <-\mu_{A} \mu_{B} \phi_{B}+\mu_{A B} \mu_{B}\left(\mu_{B} R\left(p^{m}\right)-\phi_{B}\right)+\phi_{B}\left(1-\mu_{B}\right)\left(1-\mu_{A}\right) \\
0 & <\mu_{A B}\left(\phi_{B}+\mu_{B}\left(\mu_{B} R\left(p^{m}\right)-\phi_{B}\right)\right)
\end{aligned}
$$

which is always satisfied. We note that when $\mu_{A B}=0$, these two conditions are identical.

It remains to show that the price distributions are increasing in $p$. This is readily seen for $F_{A}(p)$ and $F_{M}(p)$; for $F_{B}(p)$, this follows from the fact that $\lambda_{A} \mu_{B}-\lambda_{B}\left(1-\mu_{A}\right)>0$, as shown above. This completes the proof of Proposition 4.

Proof of Proposition 5: We start proving part (1). Recall that $\lambda_{A}=\frac{\phi_{B}-\phi_{A} \mu_{B}}{\mu_{B}^{2} R\left(p^{m}\right)+\phi_{B}\left(1-\mu_{B}\right)}$ and $\lambda_{B}=\frac{\phi_{A}}{\mu_{A} R\left(p^{m}\right)}$. Let, as above, $\phi_{A}=\gamma R\left(p^{m}\right)$ and $\phi_{B}=\beta \phi_{A}$ with $\gamma \in\left(0, \mu_{A}\right)$ and $\beta \in\left[\mu_{B} / \mu_{A}, \mu_{B} / \gamma\right)$. First, keeping $\mu_{B}$ constant, $\lambda_{A}$ does not depend on $\mu_{A B}$ and therefore $\frac{\partial \lambda_{A}}{\partial \mu_{A B}}=0$. Second, an increase in $\mu_{A B}$ lowers $\mu_{A}$ and therefore $\frac{\partial \lambda_{B}}{\partial \mu_{A B}}>0$. From these observations, it follows that $\frac{\partial \lambda_{M}}{\partial \mu_{A B}}<0$. Third, we note that $\frac{R\left(\bar{p}_{A}\right)}{R\left(\underline{p}_{A}\right)}=\frac{1}{1-\lambda_{A}}$; since $\frac{\partial \lambda_{A}}{\partial \mu_{A B}}=0$ it follows that $\frac{\partial\left(R\left(\bar{p}_{A}\right) / R\left(\underline{p}_{A}\right)\right)}{\partial \mu_{A B}}=0$. Thus, an increase in $\mu_{A B}$ leaves $\sigma_{A}$ unaltered. Fourth, we note that $R\left(\underline{p}_{B}\right)=\left(\frac{\lambda_{A} \mu_{B}-\lambda_{B}\left(\mu_{B}+\mu_{A B}\right)}{\lambda_{A} \mu_{B}}\right) R\left(p^{m}\right)$; since $\frac{\partial \lambda_{A}}{\partial \mu_{A B}}=0$ and $\frac{\partial \lambda_{B}}{\partial \mu_{A B}}>0$, it follows that an increase in $\mu_{A B}$ lowers $\underline{p}_{B}$ and therefore widens $\sigma_{B}$. Fifth, since $\underline{p}_{M}=\frac{\lambda_{A} \mu_{B} R\left(p^{m}\right)+\phi_{A}-\phi_{B}}{\left(1-\lambda_{A}\right)\left(1-\mu_{B}\right)}$ does not depend on $\mu_{A B}$, it follows that an increase in $\mu_{A B}$ leaves $\sigma_{M}$ unaltered. Finally, we note that equilibrium expected profit is $E \pi_{1}\left(\lambda_{B}=1, p^{m} ; s^{2}\right)=$ $\lambda_{A} \mu_{B} R\left(p^{m}\right)-\phi_{A}$ which does not depend on $\mu_{A B}$ and $\mu_{B}$ is kept constant. We now turn to part (2). First, we note that $\frac{\partial \lambda_{A}}{\partial \mu_{A B}}=\frac{\beta \gamma^{2}-\gamma \mu_{B}^{2}-\beta^{2} \gamma^{2}+2 \mu_{B} \gamma \beta}{\left(\mu_{B}^{2}+\beta \gamma\left(1-\mu_{B}\right)\right)^{2}}$. Inspection of this equation reveals that $\frac{\partial \lambda_{A}}{\partial \mu_{A B}}>0$ if $\gamma<\frac{\mu_{B}\left(2 \beta-\mu_{B}\right)}{\beta(\beta-1)}$. We now show that this last inequality holds in equilibrium. Indeed, $\lambda_{A}<1$ if and only if $\gamma<\frac{\mu_{B}}{\beta-1}$ and since $\gamma>0$ then $\beta>1$, so if $\frac{\mu_{B}}{\beta-1}<\frac{\mu_{B}\left(2 \beta-\mu_{B}\right)}{\beta(\beta-1)}$ then the proof follows. Note that $\frac{\mu_{B}}{\beta-1}<\frac{\mu_{B}\left(2 \beta-\mu_{B}\right)}{\beta(\beta-1)}$ whenever $\beta-\mu_{B}>0$ which is always satisfied in equilibrium. Thus, $\frac{\partial \lambda_{A}}{\partial \mu_{A B}}>0$. Second, since $\mu_{A}$ is constant, $\lambda_{B}$ does not depend on $\mu_{A B}$ and therefore $\frac{\partial \lambda_{B}}{\partial \mu_{A B}}=0$. As a consequence $\frac{\partial \lambda_{M}}{\partial \mu_{A B}}<0$. Third, since $R\left(\underline{p}_{A}\right)=\left(1-\lambda_{A}\right) R\left(\bar{p}_{A}\right)$, it follows that $\frac{R\left(\bar{p}_{A}\right)}{R\left(\underline{p}_{A}\right)}=\frac{1}{1-\lambda_{A}}$; thus since $\frac{\partial \lambda_{A}}{\partial \mu_{A B}}>0$ it follows that an increase in $\mu_{A B}$ increases $\lambda_{A}$, which also implies that it increases $\frac{R\left(\bar{p}_{A}\right)}{R\left(\underline{p}_{A}\right)}$ and therefore widens $\sigma_{A}$. Fourth, since $R\left(\underline{p}_{M}\right)=\frac{\lambda_{A}\left(1-\mu_{A}-\mu_{A B}\right) R\left(p^{m}\right)+\phi_{A}-\phi_{B}}{\left(1-\lambda_{A}\right)\left(\mu_{A}+\mu_{A B}\right)}$, we 
can calculate:

$$
\frac{\partial R\left(\underline{p}_{M}\right)}{\partial \mu_{A B}}=\frac{\frac{\partial \lambda_{A}}{\partial \mu_{A B}}\left(1-\mu_{B}\right)\left(\mu_{B} R\left(p^{m}\right)+\phi_{A}-\phi_{B}\right)-\left(1-\lambda_{A}\right)\left(\lambda_{A} R\left(p^{m}\right)+\phi_{A}-\phi_{B}\right)}{\left(1-\lambda_{A}\right)^{2}\left(1-\mu_{B}\right)^{2}}
$$

Inspection of this derivative reveals that $\partial R\left(\underline{p}_{M}\right) / \partial \mu_{A B}>0$ if and only if its numerator is positive. Using the expressions for $\frac{\partial \lambda_{A}}{\partial \mu_{A B}}$ and $\lambda_{A}$ we obtain that the condition above is satisfied if and only if $\left(\mu_{B}-\gamma(\beta-1)\right)^{2}\left(1-\mu_{B}\right)>0$, which is always satisfied. Thus, an increase in $\mu_{A B}$ narrows $\sigma_{M}$. Fifth, using the expression for $R\left(\underline{p}_{B}\right)$ we can derive:

$$
\frac{\partial R\left(\underline{p}_{B}\right)}{\partial \mu_{A B}}=\frac{\lambda_{B}\left(1-\mu_{A}\right)}{\lambda_{A}^{2} \mu_{B}^{2}}\left(\mu_{B} \frac{\partial \lambda_{A}}{\partial \mu_{A B}}-\lambda_{A}\right)
$$

Using the expressions for $\frac{\partial \lambda_{A}}{\partial \mu_{A B}}$ and $\lambda_{A}$ we obtain that

$$
\frac{\partial R\left(\underline{p}_{B}\right)}{\partial \mu_{A B}}=\frac{\lambda_{B}\left(1-\mu_{A}\right)\left(\gamma\left(2 \mu_{B}-\beta-\mu_{B}^{2}\right)+\mu_{B}^{2}\right)}{\lambda_{A}^{2} \mu_{B}^{2}}
$$

We now prove that $2 \mu_{B}-\beta-\mu_{B}^{2} \leq 0$. Suppose, on the contrary, that $2 \mu_{B}-\beta-\mu_{B}^{2}>0$ or $\beta<\mu_{B}\left(2-\mu_{B}\right)$; since in equilibrium $\lambda_{A}<1$ if and only if $\gamma<\frac{\mu_{B}}{\beta-1}$ and $\beta>1$, we must require that $\mu_{B}\left(2-\mu_{B}\right)>1$, which is never satisfied. As a consequence $\frac{\partial R\left(\underline{p}_{B}\right)}{\partial \mu_{A B}} \geq 0$ if and only if $\gamma \leq \frac{\mu_{B}^{2}}{\beta-\mu_{B}\left(2-\mu_{B}\right)}=\bar{\gamma}$, otherwise $\frac{\partial R\left(\underline{p}_{B}\right)}{\partial \mu_{A B}}<0$. Finally, we observe that firm equilibrium profits change with $\mu_{A B}$ as follows: $\frac{\partial E \pi_{i}\left(\lambda_{B}=1, p^{m} ; s^{-i}\right)}{\partial \mu_{A B}}=R\left(p^{m}\right)\left(\mu_{B} \frac{\partial \lambda_{A}}{\partial \mu_{A B}}-\lambda_{A}\right)$. Using the previous arguments, it follows that $\frac{\partial E \pi_{i}\left(\lambda_{B}=1, p^{m} ; s^{-i}\right)}{\partial \mu_{A B}} \geq 0$ if and only if $\gamma \leq \bar{\gamma}$. We note that the region of parameters for which $\gamma \leq \bar{\gamma}$ and the positive-profits equilibrium exists is not empty. The proof is now complete.

Proof of Proposition 7: We first prove that firms cannot obtain positive profits in equilibrium. The proof borrows from some of the results above. In particular, it is readily seen that Lemma 1 also holds if firms can practise price discrimination; moreover, a positive-profits equilibrium where $\lambda_{M}=0$ does not exist (Proposition 1) when firms can practise price discrimination. We now prove that $\lambda_{A}+\lambda_{M}=1$ cannot be part of an equilibrium. Let us denote firm $i$ 's strategy as $s^{i}=\left\{\left(\lambda_{A}, F_{A}(p)\right),\left(\lambda_{M}, \widetilde{F}_{A}(p), \widetilde{F}_{B}(p)\right)\right\}, \sigma_{A}, \widetilde{\sigma}_{A}$ and $\widetilde{\sigma}_{B}$ be the supports of the price distributions, and $\bar{p}_{A}, \widetilde{\bar{p}}_{A}$ and $\widetilde{\bar{p}}_{B}$ the upper bounds of the supports. We note that $F_{A}(p), \widetilde{F}_{A}(p)$ and $\widetilde{F}_{B}(p)$ must 
be atomless. The profits to a firm advertising in segment $A$ would be:

$$
E \pi_{i}\left(\lambda_{A}=1, p \in \sigma_{A} ; s^{-i}\right)=R(p) \mu_{A}\left[\lambda_{A}\left(1-F_{A}(p)\right)+\lambda_{M}\left(1-\widetilde{F}_{A}(p)\right)\right]-\phi_{A} .
$$

Likewise, the profits to a firm advertising in the entire market would be:

$$
\begin{aligned}
E \pi_{i}\left(\lambda_{M}=1, p_{A} \in \widetilde{\sigma}_{A}, p_{B} \in \widetilde{\sigma}_{B} ; s^{-i}\right)= & \lambda_{A} R\left(p_{B}\right) \mu_{B}+\lambda_{A} \mu_{A} R\left(p_{A}\right)\left(1-F_{A}\left(p_{A}\right)\right)+ \\
& \lambda_{M} \mu_{A} R\left(p_{A}\right)\left(1-\widetilde{F}_{A}\left(p_{A}\right)\right)+\lambda_{M} \mu_{B} R\left(p_{B}\right)\left(1-\widetilde{F}_{B}\left(p_{B}\right)\right) \\
& -\phi_{A}-\phi_{B} .
\end{aligned}
$$

We note that $\bar{p}_{A}<\widetilde{\bar{p}}_{A}$ otherwise a firm charging $\bar{p}_{A}$ in $A$ would make negative profits. This implies that $\widetilde{\bar{p}}_{A}=p^{m}$. It must also be the case that $\widetilde{\bar{p}}_{B}=p^{m}$. Since $p^{m} \in \widetilde{\sigma}_{A} \cap \widetilde{\sigma}_{B}$, the proof now follows that of Proposition 1. The case $\lambda_{B}+\lambda_{M}=1$ is ruled out similarly.

It remains to prove that firms cannot make positive profits when $\lambda_{j}>0, j=A, B, M$. Let $s^{i}=$ $\left\{\left(\lambda_{A}, F_{A}(p)\right),\left(\lambda_{B}, F_{B}(p)\right),\left(\lambda_{M}, \widetilde{F}_{A}(p), \widetilde{F}_{B}(p)\right)\right\}$ denote firm $i$ 's strategy, $\sigma_{A}, \sigma_{B}, \widetilde{\sigma}_{A}$ and $\widetilde{\sigma}_{B}$ be the supports of the price distributions, and $\bar{p}_{A}, \bar{p}_{B}, \widetilde{\bar{p}}_{A}$ and $\widetilde{\bar{p}}_{B}$ the upper bounds of the supports. We can write down the payoff to a firm from the different advertising strategies:

$$
\begin{aligned}
E \pi_{i}\left(\lambda_{A}=1, p \in \sigma_{A} ; s^{-i}\right) & =R(p) \mu_{A}\left[\lambda_{A}\left(1-F_{A}(p)\right)+\lambda_{B}+\lambda_{M}\left(1-\widetilde{F}_{A}(p)\right)\right]-\phi_{A} \\
E \pi_{i}\left(\lambda_{B}=1, p \in \sigma_{B} ; s^{-i}\right) & =R(p) \mu_{B}\left[\lambda_{A}+\lambda_{B}\left(1-F_{B}(p)\right)+\lambda_{M} p\left(1-\widetilde{F}_{B}(p)\right)\right]-\phi_{B} \\
E \pi_{i}\left(\lambda_{M}=1, p_{A} \in \widetilde{\sigma}_{A}, p_{B} \in \widetilde{\sigma}_{B} ; s^{-i}\right) & =E \pi_{i}\left(\lambda_{A}=1, p_{A} \in \widetilde{\sigma}_{A} ; s^{-i}\right)+E \pi_{i}\left(\lambda_{B}=1, p_{B} \in \widetilde{\sigma}_{B} ; s^{-i}\right)
\end{aligned}
$$

We note first that $F_{A}(p), F_{B}(p), \widetilde{F}_{A}(p)$ and $\widetilde{F}_{B}(p)$ must be atomless. We now note that $\sigma_{A} \cap \widetilde{\sigma}_{A}$ cannot be empty. Otherwise, e.g. if $\bar{p}_{A}<\underline{\underline{p}}_{A}$ a firm advertising $\bar{p}_{A}$ in segment $A$ would gain by increasing its price; if, instead $\widetilde{\bar{p}}_{A}<\underline{p}_{A}$, a firm advertising $\widetilde{\bar{p}}_{A}$ in segment $A$ and $p_{B} \in \widetilde{\sigma}_{B}$ in segment $B$ at the same time would gain by increasing the price advertised in segment $A$. The same arguments imply that $\sigma_{B} \cap \widetilde{\sigma}_{B}$ cannot be empty. Now let $p_{1} \in \sigma_{A} \cap \widetilde{\sigma}_{A}$ and $p_{2} \in \sigma_{B} \cap \widetilde{\sigma}_{B}$. Since the firms must be indifferent between advertising $p_{1}$ in segment $A, p_{2}$ in segment $B$ and $\left(p_{1}, p_{2}\right)$ in the entire market, this implies that firms profits must be zero. This completes the proof that firms cannot obtain positive profits in equilibrium. The equilibrium characterization offered in the result 
readily follows from an elaboration of Theorem 1 . The proof of the other zero-profits equilibria is available from the authors upon request.

\section{References}

[1] Armstrong, M. and J. Vickers (2001), "Competitive Price Discrimination," Rand Journal of Economics 32-4, 579-605.

[2] d'Aspremont, C., J. J. Gabszewicz and J.-F. Thisse (1979), "On Hotelling's Stability in Competition", Econometrica 47, 1145-1150.

[3] Bester, H. and E. Petrakis (1995), "Price Competition and Advertising in Oligopoly", European Economic Review, 39, 1075-1088.

[4] Butters, G. (1977), "Equilibrium Distributions of Sales and Advertising Prices", Review of Economic Studies, 44, 465-491.

[5] Esteban, L., Gil, A. and J. M. Hernández (2001), "Informative Advertising and Optimal Targeting in a Monopoly," Journal of Industrial Economics 49, 161-180.

[6] Grossman G. and C. Shapiro (1984), "Informative Advertising with Differentiated Products," Review of Economic Studies 51, 63-82.

[7] Holmes, T. (1989), "The Effects of Third-Degree Price Discrimination in Oligopoly," American Economic Review 79, 244-250.

[8] Iyer, G., D. Soberman and J. M. Villas-Boas (2002), "The Targeting of Advertising," mimeo.

[9] Rosenthal, R. W. (1980), "A Model in Which an Increase in the Number of Sellers Leads to a Higher Price," Econometrica 48, 1575-79.

[10] Roy, S. (2000), "Strategic Segmentation of a Market," International Journal of Industrial Organization 18-8, 1279-1290.

[11] Shaked, A. and J. Sutton (1982), "Relaxing Price Competition through Product Differentiation," Review of Economic Studies 51(5), 1469-1483. 
[12] Sharkey, W. W. and D. S. Sibley (1993), "A Bertrand model of pricing and entry," Economics Letters, 41-2, 199-206.

[13] Stahl, D. O. (1994), "Oligopolistic Pricing and Advertising," Journal of Economic Theory 64, 162-177.

[14] Stegeman, M. (1991), "Advertising in Competitive Markets", American Economic Review 81, 210-223.

[15] Stole, L. (2003), "Price Discrimination and Imperfect Competition," prepared for the Handbook of Industrial Organization.

[16] Thisse, J.-F. and X. Vives (1988), "On the Strategic Choice of Spatial Price Policy," American Economic Review 78, 122-137. 


\section{CESifo Working Paper Series}

(for full list see www.cesifo.de)

1132 David M. Newbery, Privatising Network Industries, February 2004

1133 Charles Yuji Horioka, The Stagnation of Household Consumption in Japan, February 2004

1134 Eiji Fujii, Exchange Rate Pass-Through in the Deflationary Japan: How Effective is the Yen's Depreciation for Fighting Deflation?, February 2004

1135 Mark M. Spiegel and Nobuyoshi Yamori, Determinants of Voluntary Bank Disclosure: Evidence from Japanese Shinkin Banks, Febrary 2004

1136 Robert Dekle and Kenneth Kletzer, Deposit Insurance, Regulatory Forbearance and Economic Growth: Implications for the Japanese Banking Crisis, February 2004

1137 Takatoshi Ito and Kimie Harada, Bank Fragility in Japan, 1995-2003, February 2004

1138 Kunio Okina and Shigenori Shiratsuka, Policy Duration Effect under Zero Interest Rates: An Application of Wavelet Analysis, February 2004

1139 Francine D. Blau and Lawrence M. Kahn, Do Cognitive Test Scores Explain Higher U.S. Wage Inequality?, February 2004

1140 Michael Rauscher, Economic Growth and Tax-Competing Leviathans, February 2004

1141 Ernst Fehr and Jean-Robert Tyran, Money Illusion and Coordination Failure, February 2004

1142 Ingo Vogelsang, Network Utilities in the U.S. - Sector Reforms without Privatization, March 2004

1143 Marc-Andreas Muendler, Estimating Production Functions When Productivity Change is Endogenous, March 2004

1144 Sascha O. Becker, Samuel Bentolila, Ana Fernandes, and Andrea Ichino, Job Insecurity and Children's Emancipation, March 2004

1145 Pascalis Raimondos-Møller and Alan D. Woodland, Non-Preferential Trading Clubs, March 2004

1146 Robert Fenge and Matthias Wrede, EU Regional Policy: Vertical Fiscal Externalities and Matching Grants, March 2004

1147 Chi-Yung Ng and John Whalley, Geographical Extension of Free Trade Zones as Trade Liberalization: A Numerical Simulation Approach, March 2004 
1148 Marc-Andreas Muendler, Trade, Technology, and Productivity: A Study of Brazilian Manufacturers, 1986-1998, March 2004

1149 Eugene Beaulieu, Vivek H. Dehejia, and Hazrat-Omar Zakhilwal, International Trade, Labour Turnover, and the Wage Premium: Testing the Bhagwati-Dehejia Hypothesis for Canada, March 2004

1150 Giorgio Brunello and Francesca Gambarotto, Agglomeration Effects on EmployerProvided Training: Evidence from the UK, March 2004

1151 S. Brock Blomberg, Gregory D. Hess, and Athanasios Orphanides, The Macroeconomic Consequences of Terrorism, March 2004

1152 Bodo Sturm and Joachim Weimann, Unilateral Emissions Abatement: An Experiment, March 2004

1153 Wolfgang Ochel, Welfare-to-Work Experiences with Specific Work-First Programmes in Selected Countries, March 2004

1154 Jan K. Brueckner and Eric Pels, European Airline Mergers, Alliance Consolidation, and Consumer Welfare, March 2004

1155 Aaron Tornell, Frank Westermann, and Lorenza Martínez, NAFTA and Mexico's Economic Performance, March 2004

1156 George Economides, Sarantis Kalyvitis, and Apostolis Philippopoulos, Do Foreign Aid Transfers Distort Incentives and Hurt Growth? Theory and Evidence from 75 Aid-recipient Countries, March 2004

1157 Robert Fenge and Volker Meier, Are Family Allowances and Fertility-related pensions Siamese Twins?, March 2004

1158 Bruno S. Frey, Simon Luechinger, and Alois Stutzer, Valuing Public Goods: The Life Satisfation Approach, March 2004

1159 Jerome L. Stein and Guay C. Lim, Asian Crises: Theory, Evidence, Warning-Signals, March 2004

1160 Romain Ranciere, Aaron Tornell, and Frank Westermann, Crises and Growth: A ReEvaluation, March 2004

1161 Assaf Razin and Efraim Sadka, Transparency, Specialization and FDI, March 2004

1162 Ludger Woessmann, How Equal Are Educational Opportunities? Family Background and Student Achievement in Europe and the United States, March 2004

1163 B.M.S. van Praag and Barbara E. Baarsma, Using Happiness Surveys to Value Intangibles: The Case of Airport Noise, March 2004

1164 Aaron Tornell, Frank Westermann, and Lorenza Martínez, The Positive Link Between Financial Liberalization, Growth, and Crises, March 2004 
1165 Helge Berger and Carsten Hefeker, One Country, One Vote? Labor Market Structure and Voting Rights in the ECB, March 2004

1166 Clemens Fuest and Martin Kolmar, A Theory of User-Fee Competition, March 2004

1167 Friedrich Schneider and Robert Klinglmair, Shadow Economies around the World: What Do We Know?, April 2004

1168 Horst Raff and Nicolas Schmitt, Exclusive Dealing and Common Agency in International Markets, April 2004

1169 M. Hashem Pesaran and Allan Timmermann, Real Time Econometrics, April 2004

1170 Sean D. Barrett, Privatisation in Ireland, April 2004

1171 V. Anton Muscatelli, Patrizio Tirelli and Carmine Trecroci, Can Fiscal Policy Help Macroeconomic Stabilisation? Evidence from a New Keynesian Model with Liquidity Constraints, April 2004

1172 Bernd Huber and Marco Runkel, Tax Competition, Excludable Public Goods and User Charges, April 2004

1173 John McMillan and Pablo Zoido, How to Subvert Democracy: Montesinos in Peru, April 2004

1174 Theo Eicher and Jong Woo Kang, Trade, Foreign Direct Investment or Acquisition: Optimal Entry Modes for Multinationals, April 2004

1175 Chang Woon Nam and Doina Maria Radulescu, Types of Tax Concessions for Attracting Foreign Direct Investment in Free Economic Zones, April 2004

1176 M. Hashem Pesaran and Andreas Pick, Econometric Issues in the Analysis of Contagion, April 2004

1177 Steinar Holden and Fredrik Wulfsberg, Downward Nominal Wage Rigidity in Europe, April 2004

1178 Stefan Lachenmaier and Ludger Woessmann, Does Innovation Cause Exports? Evidence from Exogenous Innovation Impulses and Obstacles, April 2004

1179 Thiess Buettner and Johannes Rincke, Labor Market Effects of Economic Integration The Impact of Re-Unification in German Border Regions, April 2004

1180 Marko Koethenbuerger, Leviathans, Federal Transfers, and the Cartelization Hypothesis, April 2004

1181 Michael Hoel, Tor Iversen, Tore Nilssen, and Jon Vislie, Genetic Testing and Repulsion from Chance, April 2004 
1182 Paul De Grauwe and Gunther Schnabl, Exchange Rate Regimes and Macroeconomic Stability in Central and Eastern Europe, April 2004

1183 Arjan M. Lejour and Ruud A. de Mooij, Turkish Delight - Does Turkey's accession to the EU bring economic benefits?, May 2004

1184 Anzelika Zaiceva, Implications of EU Accession for International Migration: An Assessment of Potential Migration Pressure, May 2004

1185 Udo Kreickemeier, Fair Wages and Human Capital Accumulation in a Global Economy, May 2004

1186 Jean-Pierre Ponssard, Rent Dissipation in Repeated Entry Games: Some New Results, May 2004

1187 Pablo Arocena, Privatisation Policy in Spain: Stuck Between Liberalisation and the Protection of Nationals' Interests, May 2004

1188 Günter Knieps, Privatisation of Network Industries in Germany: A Disaggregated Approach, May 2004

1189 Robert J. Gary-Bobo and Alain Trannoy, Efficient Tuition Fees, Examinations, and Subsidies, May 2004

1190 Saku Aura and Gregory D. Hess, What's in a Name?, May 2004

1191 Sjur Didrik Flåm and Yuri Ermoliev, Investment Uncertainty, and Production Games, May 2004

1192 Yin-Wong Cheung and Jude Yuen, The Suitability of a Greater China Currency Union, May 2004

1193 Inés Macho-Stadler and David Pérez-Castrillo, Optimal Enforcement Policy and Firms' Emissions and Compliance with Environmental Taxes, May 2004

1194 Paul De Grauwe and Marianna Grimaldi, Bubbles and Crashes in a Behavioural Finance Model, May 2004

1195 Michel Berne and Gérard Pogorel, Privatization Experiences in France, May 2004

1196 Andrea Galeotti and José Luis Moraga-González, A Model of Strategic Targeted Advertising, May 2004 